Czado, Delwarde, Denuit:

\title{
Bayesian Poisson Log-Bilinear Mortality Projections
}

Sonderforschungsbereich 386, Paper 398 (2004)

Online unter: http://epub.ub.uni-muenchen.de/

Projektpartner
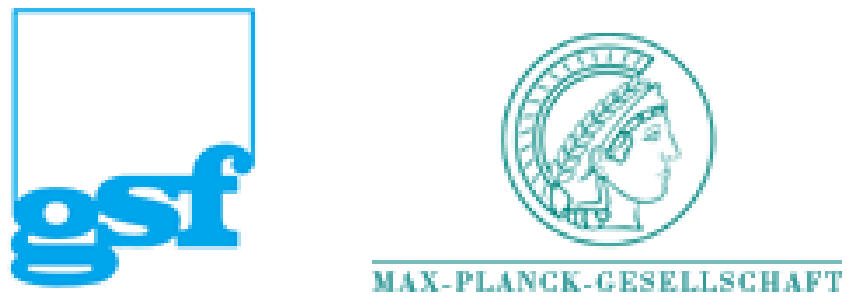


\section{BAYESIAN POISSON LOG-BILINEAR MORTALITY PROJECTIONS}

\section{Claudia Czado ${ }^{\dagger}$, Antoine DelWarde $^{\ddagger} \&$ Michel Denuit $*, \ddagger$}

${ }^{\dagger}$ SCA Zentrum Mathematik

Technische Universität München

D-85748 Garching bei Munich, Germany

†nstitut des Sciences Actuarielles

Université Catholique de Louvain

B-1348 Louvain-la-Neuve, Belgium

*Institut de Statistique

Université Catholique de Louvain

B-1348 Louvain-la-Neuve, Belgium

September 8, 2004 


\begin{abstract}
Mortality projections are major concerns for public policy, social security and private insurance. This paper implements a Bayesian log-bilinear Poisson regression model to forecast mortality. Computations are carried out using Markov Chain Monte Carlo methods in which the degree of smoothing is learnt from the data. Comparisons are made with the approach proposed by Brouhns, Denuit \& Vermunt (2002a,b), as well as with the original model of LEE \& CARTER (1992).
\end{abstract}

Key words and phrases: projected lifetables, expected remaining lifetimes, Poisson regression, MCMC. 


\section{Introduction}

\subsection{Lee-Carter model for mortality projections}

Mortality forecasts are used in a wide variety of fields: for health policy making, for directing pharmaceutical research, social security, for retirement fund planning and for life insurance, to name just a few. During the 20th century, it is now well documented that the human mortality globally declined: in most industrialized countries, mortality at adult and old ages reveals decreasing annual death probabilities.

In this paper, we analyze the changes in mortality as a function of both age $x$ and calendar time $t$. Henceforth, $\mu_{x}(t)$ will denote the force of mortality at age $x$ and time $t$. Throughout this paper, we assume that given any integer age $x$ and calendar year $t$,

$$
\mu_{x+\xi}(t+\tau)=\mu_{x}(t) \text { for } 0 \leq \xi, \tau<1 .
$$

This is best illustrated with the aid of a coordinate system that has calendar time as abscissa and age as coordinate. Such a representation is called a Lexis diagram after the German demographer who introduced it. Both time scales are divided into yearly bands, which partition the Lexis plane into square segments. Model (1.1) assumes that the mortality rate is constant within each square, but allows it to vary between squares. We denote as $D_{x t}$ the number of deaths recorded at age $x$ during year $t$, from an exposure-to-risk $E_{x t}$ (that is, $E_{x t}$ is the number of person-years from which $D_{x t}$ occurred).

A powerful and elegant approach to mortality forecasts has been pioneered by LEE \& CARTER (1992). Those authors proposed a remarkably simple model for mortality projections, specifying a log-bilinear form for the force of mortality $\mu_{x}(t)$. The method is in essence a relational model

$$
\ln \widehat{\mu_{x}}(t)=\alpha_{x}+\beta_{x} \kappa_{t}+\epsilon_{x}(t)
$$

where $\widehat{\mu_{x}}(t)=D_{x t} / E_{x t}$ denotes the observed force of mortality at age $x$ during year $t$, the $\epsilon_{x}(t)$ 's are homoskedastic centered error terms and where the parameters are subject to the constraints

$$
\sum_{t} \kappa_{t}=0 \text { and } \sum_{x} \beta_{x}=1
$$

ensuring model identification.

An important aspect of Lee-Carter methodology is that the time factor $\kappa_{t}$ is intrinsically viewed as a stochastic process. Box-Jenkins techniques are then used to estimate and forecast $\kappa_{t}$ within an ARIMA time series model. From this forecast of the general level of mortality, the actual age-specific rates are derived using the estimated age effects. This in turn yields projected life expectancies.

For a review of recent applications of the Lee-Carter methodology, we refer the interested readers to LEE (2000). It is worth to mention that the Lee-Carter model is used by the US Census Bureau as a benchmark for their population forecasts, and its use has been recommended by the US Social Security Technical Advisory Panels. It appears to be the determinant method in the literature. 


\subsection{Poisson log-bilinear model for mortality projections}

According to Brillinger (1986) and Alho (2000), the Poisson approximation for the number of deaths occurring in a square of the Lexis diagram is plausible. This lead Sithole, Haberman \& Verrall (2000) and Renshaw \& Haberman (2003a,b) to implement an alternative approach to mortality forecasting: calendar time enters the model as a known covariate and a regression model based on heteroskedastic Poisson error structures is used.

A closely related model has been proposed by Brounns, Denuit \& Vermunt (2002a,b), keeping the Lee-Carter log-bilinear form for the forces of mortality. Specifically, Brounns ET AL. (2002a,b) considered that

$$
D_{x t} \sim \operatorname{Poisson}\left(E_{x t} \mu_{x}(t)\right) \text { with } \mu_{x}(t)=\exp \left(\alpha_{x}+\beta_{x} \kappa_{t}\right)
$$

where the parameters are still subjected to the constraints (1.3).

There is thus a key difference between RENSHAw \& HABERMAN (2003a) and Brouhns ET AL. (2002b) that centres on the intepretation of time: in BROUHNS ET AL. (2002b) time is modeled as a factor and under the approach proposed by RENSHAW \& HABERMAN (2003) is modelled as a known covariate. We believe that the former approach is preferable since we do not constrain ex ante the effect of calendar time to some known functional form.

Instead of resorting to SVD for estimating $\alpha_{x}, \beta_{x}$ and $\kappa_{t}$, BROUHns ET AL. (2002a,b) estimated the parameters by maximizing the log-likelihood based on model (1.4). As in the Lee-Carter approach, ARIMA models are then used to forecast the $\kappa_{t}$ 's.

\subsection{Scope of the paper}

In all the papers mentioned above, the modelling still proceeds in two steps: first the mortality index $\kappa_{t}$ is estimated and then it is extrapolated using Box-Jenkins methodology. Possible incoherence may arise from this two-step procedure. In order to avoid this flaw, we purpose to integrate both steps into a Bayesian model. Bayesian formulations assume some sort of smoothness of age and period effects in order to improve estimation and facilitate prediction. Intuitively, we expect smooth variations of the mortality rates over the Lexis plane. In order to implement this idea, we resort to a Bayesian model in which the prior portion imposes smoothness by relating the underlying mortality rates to each other over the Lexis plane. As a consequence, the rate estimate in each age-year square "borrows strength" from information in adjacent squares. An important advantage of incorporating the idea of smoothness is that it is possible to use the model for purposes of forecasting future mortality rates.

The Bayesian modelling treats all unknown parameters $\alpha_{x}, \beta_{x}$ and $\kappa_{t}$ as random variables and derives their distribution conditional upon the known information $\left(E_{x t}, D_{x t}\right)$. Until recently, fully Bayesian analyses had been computationally infeasible and approximation methods were often utilized instead. This changed in the early 1990's with computer-intensive Markov Chain Monte Carlo (MCMC) simulation methods (see CHIB (2001) for a summary and Gilks ET AL. (1996) for applications). The Monte Carlo approach allows for inference based on sampling the posterior distribution of the parameters. A particularly attractive feature of this approach is the ease with which we can then explore the uncertainty associated with the estimates and the forecasts. 
A Bayesian treatment of mortality projections has been proposed by GIROsi \& KING (2003). The approach followed by these authors is nevertheless entirely different from the one adopted in this paper. We refer the reader to the interesting monograph written by these authors for more details.

\subsection{Agenda}

Section 2 describes the model and details the prior assumption on each set of parameters. Section 3 derives the MCMC algorithm yielding the a posteriori distribution of the parameters. A numerical illustration is discussed in Section 4, where the results obtained with the methodology developed in this paper are compared with former ones.

By convention, vectors and matrices are denoted by bold lower and upper cases, respectively. Parameters and hyper-parameters are denoted by Greek letters. All the vectors are assumed to be column vectors and the superscript' indicates transposition. We denote as $x_{\min }, x_{\min }+1, \ldots, x_{\max }$ the observed age range and as $t_{\min }, t_{\min }+1, \ldots, t_{\max }$ the observed calendar time range. Moreover, $M=x_{\max }-x_{\min }+1$ is the number of different ages considered in the model, and $T=t_{\max }-t_{\min }+1$ is the number of calendar years. We denote as $\boldsymbol{I}_{M}$ (resp. $\boldsymbol{I}_{T}$ ) the $M$-dimensional (resp. $T$-dimensional) identity matrix. Further, $X \sim \mathcal{N} \operatorname{ormal}\left(m, \sigma^{2}\right)$ indicates that the random variable $X$ is normally distributed with mean $m$ and variance $\sigma^{2}$, while $\boldsymbol{X} \sim \mathcal{N}_{\text {ormal }}(\boldsymbol{m}, \boldsymbol{\Sigma})$ indicates that the random vector $\boldsymbol{X}$ is normally distributed with mean vector $\boldsymbol{m}$ and variance-covariance matrix $\boldsymbol{\Sigma}$.

\section{Model and prior distributions}

\subsection{Likelihood function}

Let us consider the Poisson log-bilinear model (1.4) supplemented with the constraints (1.3) in order to ensure the identifiability of the model. This model comprises three sets of parameters: $\boldsymbol{\alpha}=\left(\alpha_{x_{\min }}, \ldots, \alpha_{x_{\max }}\right)^{\prime}, \boldsymbol{\beta}=\left(\beta_{x_{\min }}, \ldots, \beta_{x_{\max }}\right)^{\prime}$ and $\boldsymbol{\kappa}=\left(\kappa_{t_{\min }}, \ldots, \kappa_{t_{\max }}\right)^{\prime}$. The likelihood function associated with the data points $\left(E_{x t}, D_{x t}\right), x=x_{\min }, x_{\min }+1, \ldots, x_{\max }$ and $t=t_{\min }, t_{\min }+1, \ldots, t_{\max }$, writes

$$
\begin{aligned}
L(\boldsymbol{\alpha}, \boldsymbol{\beta}, \boldsymbol{\kappa}) & =\prod_{x} \prod_{t} \frac{\exp \left(-E_{x t} \exp \left(\alpha_{x}+\beta_{x} \kappa_{t}\right)\right)\left(E_{x t} \exp \left(\alpha_{x}+\beta_{x} \kappa_{t}\right)\right)^{D_{x t}}}{D_{x t} !} \\
& \propto \prod_{x} \prod_{t} \exp \left(-E_{x t} \exp \left(\alpha_{x}+\beta_{x} \kappa_{t}\right)+D_{x t}\left(\alpha_{x}+\beta_{x} \kappa_{t}\right)\right) .
\end{aligned}
$$

As usual, the first stage of a Bayesian analysis is to specify a prior probability density for the parameters $\boldsymbol{\alpha}, \boldsymbol{\beta}$ and $\boldsymbol{\kappa}$ involved in the Poisson log-bilinear model. This prior should support the local regularities that are believed to exist.

\subsection{Prior distribution for the time index $\kappa$}

The time index $\kappa_{t}$ represents the time trend. The actual forces of mortality change according to an overall mortality index $\kappa_{t}$ modulated by an age response $\beta_{x}$. In the Lee-Carter 
approach, as well as in its Poisson counterpart, the $\kappa_{t}$ 's are projected using an ARIMA model. In that respect, a random walk with drift was found the most appropriate for the data analyzed by LEE \& CARTER (1992). In practice, that simple model for $\kappa_{t}$ is used almost exclusively and accounts for nearly all real applications.

Our prior assumption for the time index derives from these empirical evidences. We use an autoregressive prior distribution with a linear mean for $\boldsymbol{\kappa}$. More specifically, we consider the model

$$
\kappa_{t}-\gamma_{1}-\gamma_{2} t=\rho\left(\kappa_{t-1}-\gamma_{1}-\gamma_{2}(t-1)\right)+\epsilon_{t} \text { for } t=t_{\min }, \ldots, t_{\max }
$$

with $\kappa_{t_{\min }-1}=\gamma_{1}+\gamma_{2}\left(t_{\min }-1\right)$. The errors $\epsilon_{t} \sim \mathcal{N} \operatorname{ormal}\left(0, \sigma_{\kappa}^{2}\right)$ are mutually independent. Denoting as $\eta_{t}=\gamma_{1}+\gamma_{2} t$ the linear trend for the $\kappa_{t}$ 's, (2.2) can be re-written as

$$
(\boldsymbol{\kappa}-\boldsymbol{X} \gamma)=\boldsymbol{P}(\boldsymbol{\kappa}-\boldsymbol{X} \gamma)+\boldsymbol{\epsilon}
$$

with

$$
\boldsymbol{P}=\left(\begin{array}{ccccc}
0 & \cdots & \cdots & \cdots & 0 \\
\rho & \ddots & & & \vdots \\
0 & \ddots & \ddots & & \vdots \\
\vdots & \ddots & \ddots & \ddots & \vdots \\
0 & \cdots & 0 & \rho & 0
\end{array}\right), \quad \boldsymbol{X}=\left(\begin{array}{cc}
1 & t_{\min } \\
\vdots & \vdots \\
1 & t_{\max }
\end{array}\right), \gamma=\left(\begin{array}{c}
\gamma_{1} \\
\gamma_{2}
\end{array}\right) \text { and } \boldsymbol{\epsilon} \sim \mathcal{N o r m a l}_{T}\left(\mathbf{0}, \sigma_{\kappa}^{2} \boldsymbol{I}_{\boldsymbol{T}}\right)
$$

This leads to the prior $\boldsymbol{\kappa} \sim \mathcal{N} \operatorname{ormal}_{T}\left(\boldsymbol{X} \boldsymbol{\gamma}, \sigma_{\kappa}^{2} \boldsymbol{Q}^{-1}\right)$ for the time index, with

$$
\boldsymbol{Q}=\left(\begin{array}{ccccc}
1+\rho^{2} & -\rho & 0 & \cdots & 0 \\
-\rho & 1+\rho^{2} & -\rho & \ddots & \vdots \\
0 & -\rho & \ddots & \ddots & 0 \\
\vdots & \ddots & \ddots & 1+\rho^{2} & -\rho \\
0 & \cdots & 0 & -\rho & 1
\end{array}\right)
$$

We consider the unknown $\boldsymbol{\gamma}, \rho$ and $\sigma_{\kappa}^{2}$ involved in the prior distribution of $\boldsymbol{\kappa}$ as hyperparameters. They are treated as random variables with their own prior distributions. The corresponding priors are

$$
\begin{aligned}
& \gamma \sim \mathcal{N o r m a l}_{2}\left(\boldsymbol{\gamma}_{0}, \boldsymbol{\Sigma}_{0}\right) \\
& \rho \sim \mathcal{N} \operatorname{ormal}\left(0, \sigma_{\rho}^{2}\right) \text { truncated to the interval }(0,1)
\end{aligned}
$$

and we follow the standard practice and chose an inverse gamma prior for $\sigma_{\kappa}^{2}$, that is

$$
\sigma_{\kappa}^{-2} \sim \mathcal{G} a m m a\left(a_{\kappa}, b_{\kappa}\right)
$$

With this choice, $\mathbb{E}\left[\sigma_{\kappa}^{-2}\right]=\frac{a_{\kappa}}{b_{\kappa}}$ and $\operatorname{Var}\left[\sigma_{\kappa}^{-2}\right]=\frac{a_{\kappa}}{b_{\kappa}^{2}}$. The prior distribution for the vector $\boldsymbol{\kappa}$ is thus determined by the three constants $a_{\kappa}, b_{\kappa}$ and $\sigma_{\rho}^{2}$, by the vector $\gamma_{0}$ and by the matrix $\Sigma_{0}$. 


\subsection{Prior distribution for $\beta$}

The parameters $\beta_{x}$ represent the age-specific pattern of mortality change: $\beta_{x}$ indicates the sensitivity of the logarithm of the force of mortality at age $x$ to variations in the time index. The shape of the $\beta_{x}$ profile tells which rates decline rapidly and which slowly over time in response of change in $\kappa_{t}$.

The $\beta_{x}$ profile is usually much more erratic (see BRounns ET AL. (2002a) for an illustration with Belgian data). Some of the $\beta_{x}$ 's are close to zero (especially for the ages around the accident hump, for which mortality improvements are weak, as well as for older ages) while others are quite large (around birth for instance).

Our prior assumption for the $\beta_{x}$ 's is

$$
\boldsymbol{\beta} \sim \mathcal{N}^{\circ \operatorname{rmal}_{M}}\left(\mathbf{0}, \sigma_{\beta}^{2} \boldsymbol{I}_{\boldsymbol{M}}\right) .
$$

In words, we start from the assumption that no mortality improvements occur for the population under study. The data will of course appropriately transform the prior distribution in case improvements do occur, as expected. Prior distributions for the hyperparameter $\sigma_{\beta}^{2}$ is taken to be inverse gamma, to facilitate the computation. Specifically,

$$
\sigma_{\beta}^{-2} \sim \mathcal{G a m m a}\left(a_{\beta}, b_{\beta}\right)
$$

for some constants $a_{\beta}$ and $b_{\beta}$.

\subsection{Prior distribution for $\alpha$}

For technical reasons, it is more convenient to deal with the transformed vector $\boldsymbol{e}=\exp \boldsymbol{\alpha}$.

The prior distribution for $\boldsymbol{e}$ is

$$
e_{x} \sim \mathcal{G a m m a}\left(a_{x}, b_{x}\right)
$$

for some constants $a_{x}$ and $b_{x}$, with $x=x_{\min }, \ldots, x_{\max }$.

\subsection{Summary of the model}

The model can be summarized as follows:

Numbers of deaths

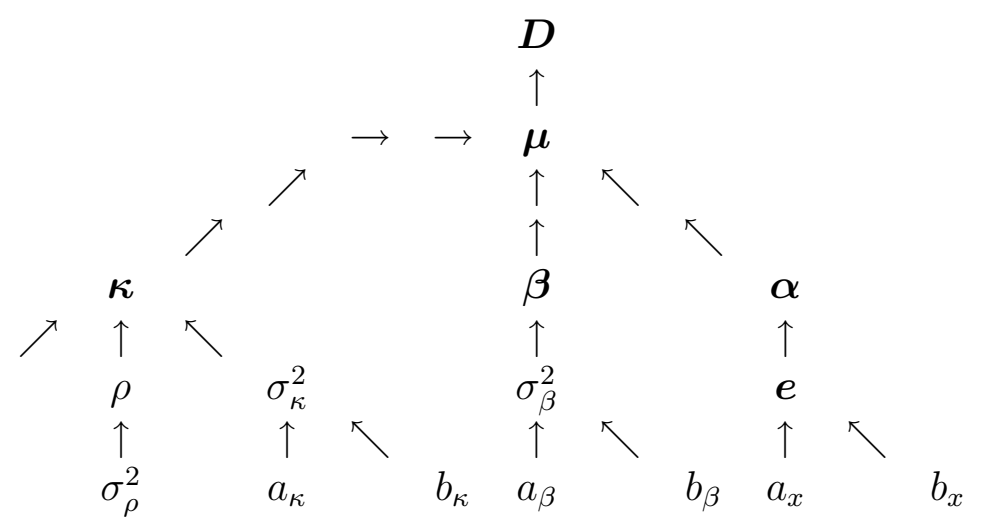

Mortality rates

Parameters

Hyperparameters

Constants $\gamma_{0}$



$\sigma_{\rho}^{2}$

$a_{\kappa} \quad b_{\kappa} \quad a_{\beta}$

$b_{x}$

The bottom line components are constants determining the distributions of the hyperparameters. The hyperparameters can be viewed on the line above. The third line represents the three sets of parameters of the Poisson log-bilinear model (1.4). 


\section{A posteriori distributions}

\subsection{MCMC approach}

Inference about the $\alpha_{x}$ 's, $\beta_{x}$ 's and $\kappa_{t}$ 's is based on the posterior density of $(\boldsymbol{\alpha}, \boldsymbol{\beta}, \boldsymbol{\kappa})$ given the mortality statistics, that is on the density

$$
p(\boldsymbol{\alpha}, \boldsymbol{\beta}, \boldsymbol{\kappa} \mid \boldsymbol{D}) \propto f(\boldsymbol{D} \mid \boldsymbol{\alpha}, \boldsymbol{\beta}, \boldsymbol{\kappa}) p(\boldsymbol{\alpha}, \boldsymbol{\beta}, \boldsymbol{\kappa}) .
$$

We make inference empirically by collecting many realizations from the posterior distribution $p(\boldsymbol{\alpha}, \boldsymbol{\beta}, \boldsymbol{\kappa} \mid \boldsymbol{D})$. This is done by simulation: it is possible to set up a Markov chain whose stationary distribution is consistent with the posterior distribution (3.1). The Monte Carlo approach allows for inference based on sampling the posterior distribution of the parameters. A particularly attractive feature of this approach is the ease with which we can then explore the uncertainty associated with the estimates and the forecasts.

In this section, we discuss implementation of the Bayes procedures via Markov Chain Monte Carlo (MCMC). In particular, we use the Gibbs sampler and Metropolis-Hastings algorithm to generate samples from the posterior (3.1).

Let us now describe the MCMC procedure more formally. Consider a random vector $\boldsymbol{Z}$ with joint probability density function $h$. In a Bayesian context, some of the components of $\boldsymbol{Z}$ are model parameters, while others may represent unobserved past or future data. Suppose $h$ is so complicated and analytically intractable that it does not permit independent random draws. In this case a MCMC simulation method may be used.

The main idea behind a MCMC method is to simulate realizations from a Markov chain which has $h$ as its stationary distribution. The resulting random draws $\boldsymbol{Z}^{(1)}, \boldsymbol{Z}^{(2)}, \ldots$ are no longer independent, but under mild regularity conditions (as described in the Appendix of

Smith \& Roberts (1993), for example), the value of $\boldsymbol{Z}^{(t)}$ tends in distribution to that of a random draw from $h$ as $t$ becomes moderately large.

Determining how long a MCMC simulation should be run is a function of the particular application. Usually, several tens of thousands of iterations are enough. In any case, the first portion of the simulated Markov chain is discarded in order to reduce the effect of the starting values. An ad hoc but useful test of convergence is obtained by running several simulations in parallel, with different starting values, and then comparing the results: the number of iterations must be increased if the results look rather different.

\subsection{Metropolis-Hastings sampling for the time index vector $\kappa$}

Metropolis-Hastings algorithms produce Markov chains whose stationary distribution is precisely (3.1) from which the sample have to be drawn. These algorithms are based on a Markov chain whose dependence on the predecessor is split into two parts: a proposal and an acceptance of the proposal. The proposals suggest an arbitrary next step in the trajectory of the chain and the acceptance makes sure the appropriate limiting direction is maintained by rejecting unwanted moves of the chain.

Let us denote as

$$
\boldsymbol{\kappa}_{-t}=\left(\kappa_{t_{\min }}, \ldots, \kappa_{t-1}, \kappa_{t+1}, \ldots, \kappa_{t_{\max }}\right)^{\prime}
$$


the time index vector $\boldsymbol{\kappa}$ without its $t$-th component. Denoting as

$$
\boldsymbol{D}_{t}=\left(D_{x_{\min }}, \ldots, D_{x_{\max }}\right)^{\prime},
$$

the vector of the $D_{x t}$ 's, we define in the same way

$$
\boldsymbol{D}_{-t}=\left(\boldsymbol{D}_{t_{\min }}, \ldots, \boldsymbol{D}_{t-1}, \boldsymbol{D}_{t+1}, \ldots, \boldsymbol{D}_{t_{\max }}\right)^{\prime}
$$

as the matrix of the death counts $D_{x t}$ without the column corresponding to calendar year $t$.

Now, each $\boldsymbol{\kappa}$ update is realized elementwise according to Metropolis-Hastings sampling. Specifically we look for the conditional probability density function $f\left(\kappa_{t} \mid \boldsymbol{\kappa}_{-t}, \boldsymbol{\alpha}, \boldsymbol{\beta}, \boldsymbol{D}, \sigma_{\kappa}^{2}, \sigma_{\beta}^{2}, \boldsymbol{\gamma}, \rho\right)$ that is, the density of $\kappa_{t}$ given all other parameters and hyper-parameters, as well as data points. Some manipulations yield

$$
\begin{aligned}
& f\left(\kappa_{t} \mid \boldsymbol{\kappa}_{-t}, \boldsymbol{\alpha}, \boldsymbol{\beta}, \boldsymbol{D}, \sigma_{\kappa}^{2}, \sigma_{\beta}^{2}, \boldsymbol{\gamma}, \rho\right) \\
= & \frac{f\left(\boldsymbol{\kappa}, \boldsymbol{\alpha}, \boldsymbol{\beta}, \boldsymbol{D}, \sigma_{\kappa}^{2}, \sigma_{\beta}^{2}, \boldsymbol{\gamma}, \rho\right)}{f\left(\boldsymbol{\kappa}_{-t}, \boldsymbol{\alpha}, \boldsymbol{\beta}, \boldsymbol{D}, \sigma_{\kappa}^{2}, \sigma_{\beta}^{2}, \boldsymbol{\gamma}, \rho\right)} \\
= & \frac{f\left(\kappa_{t_{\max }}, \boldsymbol{D}_{t_{\max }} \mid \boldsymbol{\kappa}_{-t_{\max }}, \boldsymbol{D}_{-t_{\max }}, \boldsymbol{\alpha}, \boldsymbol{\beta}, \sigma_{\kappa}^{2}, \sigma_{\beta}^{2}, \boldsymbol{\gamma}, \rho\right) f\left(\boldsymbol{\kappa}_{-t_{\max }}, \boldsymbol{D}_{-t_{\max }}, \boldsymbol{\alpha}, \boldsymbol{\beta}, \sigma_{\kappa}^{2}, \sigma_{\beta}^{2}, \boldsymbol{\gamma}, \rho\right)}{f\left(\boldsymbol{\kappa}_{-t}, \boldsymbol{D}, \boldsymbol{\alpha}, \boldsymbol{\beta}, \sigma_{\kappa}^{2}, \sigma_{\beta}^{2}, \boldsymbol{\gamma}, \rho\right)} .
\end{aligned}
$$

Iterating this formula gives

$$
\begin{aligned}
& f\left(\kappa_{t} \mid \boldsymbol{\kappa}_{-t}, \boldsymbol{\alpha}, \boldsymbol{\beta}, \boldsymbol{D}, \sigma_{\kappa}^{2}, \sigma_{\beta}^{2}, \boldsymbol{\gamma}, \rho\right) \\
= & \frac{f\left(\boldsymbol{\alpha}, \boldsymbol{\beta}, \sigma_{\kappa}^{2}, \sigma_{\beta}^{2}, \boldsymbol{\gamma}, \rho\right)}{f\left(\boldsymbol{\kappa}_{-t}, \boldsymbol{D}, \boldsymbol{\alpha}, \boldsymbol{\beta}, \sigma_{\kappa}^{2}, \sigma_{\beta}^{2}, \boldsymbol{\gamma}, \rho\right)} f\left(\kappa_{t_{\min }}, \boldsymbol{D}_{t_{\min }} \mid \boldsymbol{\alpha}, \boldsymbol{\beta}, \sigma_{\kappa}^{2}, \sigma_{\beta}^{2}, \boldsymbol{\gamma}, \rho\right) \\
& \prod_{s=t_{\min }+1}^{t_{\max }} f\left(\kappa_{s}, \boldsymbol{D}_{s} \mid \kappa_{t_{\min }}, \ldots, \kappa_{s-1}, \boldsymbol{D}_{t_{\min }}, \ldots, \boldsymbol{D}_{s-1}, \boldsymbol{\alpha}, \boldsymbol{\beta}, \sigma_{\kappa}^{2}, \sigma_{\beta}^{2}, \boldsymbol{\gamma}, \rho\right) \\
\propto & f\left(\kappa_{t_{\min }}, \boldsymbol{D}_{t_{\min }} \mid \boldsymbol{\alpha}, \boldsymbol{\beta}, \sigma_{\kappa}^{2}, \sigma_{\beta}^{2}, \boldsymbol{\gamma}, \rho\right) \\
& \prod_{s=t_{\min }+1}^{t_{\max }} f\left(\kappa_{s}, \boldsymbol{D}_{s} \mid \kappa_{t_{\min }}, \ldots, \kappa_{s-1}, \boldsymbol{D}_{t_{\min }}, \ldots, \boldsymbol{D}_{s-1}, \boldsymbol{\alpha}, \boldsymbol{\beta}, \sigma_{\kappa}^{2}, \sigma_{\beta}^{2}, \boldsymbol{\gamma}, \rho\right) .
\end{aligned}
$$

Remember that the random vectors $\boldsymbol{D}_{s}$ are mutually independent given $\boldsymbol{\kappa}, \boldsymbol{\alpha}, \boldsymbol{\beta}, \sigma_{\kappa}^{2}, \sigma_{\beta}^{2}, \boldsymbol{\gamma}, \rho$. Moreover, their conditional distribution only depends on $\left(\kappa_{s}, \boldsymbol{\alpha}, \boldsymbol{\beta}\right)$. This allows us to write

$$
\begin{aligned}
& f\left(\kappa_{s}, \boldsymbol{D}_{s} \mid \kappa_{t_{\min }}, \ldots, \kappa_{s-1}, \boldsymbol{D}_{t_{\min }}, \ldots, \boldsymbol{D}_{s-1}, \boldsymbol{\alpha}, \boldsymbol{\beta}, \sigma_{\kappa}^{2}, \sigma_{\beta}^{2}, \boldsymbol{\gamma}, \rho\right) \\
= & f\left(\boldsymbol{D}_{s} \mid \kappa_{t_{\min }}, \ldots, \kappa_{s-1}, \kappa_{s}, \boldsymbol{D}_{t_{\min }}, \ldots, \boldsymbol{D}_{s-1}, \boldsymbol{\alpha}, \boldsymbol{\beta}, \sigma_{\kappa}^{2}, \sigma_{\beta}^{2}, \boldsymbol{\gamma}, \rho\right) \\
& f\left(\kappa_{s} \mid \kappa_{t_{\min }}, \ldots, \kappa_{s-1}, \boldsymbol{D}_{t_{\min }}, \ldots, \boldsymbol{D}_{s-1}, \boldsymbol{\alpha}, \boldsymbol{\beta}, \sigma_{\kappa}^{2}, \sigma_{\beta}^{2}, \boldsymbol{\gamma}, \rho\right) \\
= & f\left(\boldsymbol{D}_{s} \mid \kappa_{s}, \boldsymbol{\alpha}, \boldsymbol{\beta}\right) f\left(\kappa_{s} \mid \kappa_{t_{\min }}, \ldots, \kappa_{s-1}, \boldsymbol{D}_{t_{\min }}, \ldots, \boldsymbol{D}_{s-1}, \boldsymbol{\alpha}, \boldsymbol{\beta}, \sigma_{\kappa}^{2}, \sigma_{\beta}^{2}, \boldsymbol{\gamma}, \rho\right) \\
= & f\left(\boldsymbol{D}_{s} \mid \kappa_{s}, \boldsymbol{\alpha}, \boldsymbol{\beta}\right) f\left(\kappa_{s} \mid \kappa_{s-1}, \boldsymbol{\gamma}, \sigma_{\kappa}^{2}, \rho\right) .
\end{aligned}
$$

Finally we find the following expression for the conditional density of $\kappa_{t}$ :

$$
\begin{aligned}
& f\left(\kappa_{t} \mid \boldsymbol{\kappa}_{-t}, \boldsymbol{\alpha}, \boldsymbol{\beta}, \boldsymbol{D}, \sigma_{\kappa}^{2}, \sigma_{\beta}^{2}, \boldsymbol{\gamma}, \rho\right) \\
\propto & f\left(\boldsymbol{D}_{t_{\min }} \mid \kappa_{t_{\min }}, \boldsymbol{\alpha}, \boldsymbol{\beta}\right) f\left(\kappa_{t_{\min }} \mid \boldsymbol{\gamma}, \sigma_{\kappa}^{2}\right) \prod_{s=t_{\min }+1}^{t_{\max }} f\left(\boldsymbol{D}_{s} \mid \kappa_{s}, \boldsymbol{\alpha}, \boldsymbol{\beta}\right) f\left(\kappa_{s} \mid \kappa_{s-1}, \boldsymbol{\gamma}, \sigma_{\kappa}^{2}, \rho\right) .
\end{aligned}
$$

Let us now consider three cases: 
(i) if $t=t_{\min }$ then

$$
\begin{aligned}
& f\left(\kappa_{t} \mid \boldsymbol{\kappa}_{-t}, \boldsymbol{\alpha}, \boldsymbol{\beta}, \boldsymbol{D}, \sigma_{\kappa}^{2}, \sigma_{\beta}^{2}, \boldsymbol{\gamma}, \rho\right) \\
\propto & f\left(\boldsymbol{D}_{t} \mid \kappa_{t}, \boldsymbol{\alpha}, \boldsymbol{\beta}\right) f\left(\kappa_{t} \mid \boldsymbol{\gamma}, \sigma_{\kappa}^{2}\right) f\left(\kappa_{t+1} \mid \kappa_{t}, \boldsymbol{\gamma}, \sigma_{\kappa}^{2}, \rho\right) \\
\propto & \prod_{x} \exp \left(-E_{x t} \exp \left(\alpha_{x}+\beta_{x} \kappa_{t}\right)\right) \prod_{x} \exp \left(\beta_{x} \kappa_{t} D_{x t}\right) \\
& \exp \left(-\frac{1}{2 \sigma_{\kappa}^{2}}\left(\kappa_{t}-\eta_{t}\right)^{2}\right) \exp \left(-\frac{1}{2 \sigma_{\kappa}^{2}}\left(\kappa_{t-1}-\eta_{t-1}-\rho\left(\kappa_{t}-\eta_{t}\right)\right)^{2}\right) .
\end{aligned}
$$

(ii) if $t=t_{\max }$ then

$$
\begin{aligned}
& f\left(\kappa_{t} \mid \boldsymbol{\kappa}_{-t}, \boldsymbol{\alpha}, \boldsymbol{\beta}, \boldsymbol{D}, \sigma_{\kappa}^{2}, \sigma_{\beta}^{2}, \boldsymbol{\gamma}, \rho\right) \\
\propto & f\left(\boldsymbol{D}_{t} \mid \kappa_{t}, \boldsymbol{\alpha}, \boldsymbol{\beta}\right) f\left(\kappa_{t} \mid \kappa_{t-1}, \boldsymbol{\gamma}, \sigma_{\kappa}^{2}, \rho\right) \\
\propto & \prod_{x} \exp \left(-E_{x t} \exp \left(\alpha_{x}+\beta_{x} \kappa_{t}\right)\right) \prod_{x} \exp \left(\beta_{x} \kappa_{t} D_{x t}\right) \\
& \exp \left(-\frac{1}{2 \sigma_{\kappa}^{2}}\left(\kappa_{t}-\eta_{t}-\rho\left(\kappa_{t-1}-\eta_{t-1}\right)\right)^{2}\right) .
\end{aligned}
$$

(iii) if $t_{\min }<t<t_{\max }$ then

$$
\begin{aligned}
& f\left(\kappa_{t} \mid \boldsymbol{\kappa}_{-t}, \boldsymbol{\alpha}, \boldsymbol{\beta}, \boldsymbol{D}, \sigma_{\kappa}^{2}, \sigma_{\beta}^{2}, \boldsymbol{\gamma}, \rho\right) \\
\propto & f\left(\boldsymbol{D}_{t} \mid \kappa_{t}, \boldsymbol{\alpha}, \boldsymbol{\beta}\right) f\left(\kappa_{t} \mid \kappa_{t-1}, \boldsymbol{\gamma}, \sigma_{\kappa}^{2}, \rho\right) f\left(\kappa_{t+1} \mid \kappa_{t}, \boldsymbol{\gamma}, \sigma_{\kappa}^{2}, \rho\right) \\
\propto & \prod_{x} \exp \left(-E_{x t} \exp \left(\alpha_{x}+\beta_{x} \kappa_{t}\right)\right) \prod_{x} \exp \left(\beta_{x} \kappa_{t} D_{x t}\right) \\
& \exp \left(-\frac{1}{2 \sigma_{\kappa}^{2}}\left(\kappa_{t}-\eta_{t}-\rho\left(\kappa_{t-1}-\eta_{t-1}\right)\right)^{2}\right) \\
& \exp \left(-\frac{1}{2 \sigma_{\kappa}^{2}}\left(\kappa_{t+1}-\eta_{t+1}-\rho\left(\kappa_{t}-\eta_{t}\right)\right)^{2}\right) .
\end{aligned}
$$

Let us decompose iteration $(i+1)$ of Metropolis-Hastings sampling for $\kappa_{t}$ update. Suppose that we have to update the value $\kappa_{t}^{(i)}$ obtained at iteration $i$. At this stage, we have already updated the preceding kappa's, so that we have at our disposal the $\kappa_{s}^{(i+1)}$ for $s<t$. We then proceed as follows:

1. First generate a candidate $\kappa_{t}^{*}$ from the $\mathcal{N} \operatorname{ormal}\left(\kappa_{t}^{(i)}, \sigma_{t}^{2}\right)$ distribution with known variance $\sigma_{t}^{2}$.

2. Then compute acceptance probability

$$
\psi\left(\kappa_{t}^{(i)}, \kappa_{t}^{*}\right)=\min \left(1, \frac{f\left(\kappa_{t}^{*} \mid \boldsymbol{\kappa}_{-t}^{(i)}, \boldsymbol{D}, \boldsymbol{\alpha}, \boldsymbol{\beta}, \sigma_{\kappa}^{2}, \sigma_{\beta}^{2}, \boldsymbol{\gamma}, \rho\right)}{f\left(\kappa_{t}^{(i)} \mid \boldsymbol{\kappa}_{-t}^{(i)}, \boldsymbol{D}, \boldsymbol{\alpha}, \boldsymbol{\beta}, \sigma_{\kappa}^{2}, \sigma_{\beta}^{2}, \boldsymbol{\gamma}, \rho\right)}\right)
$$

where

$$
\boldsymbol{\kappa}_{-t}^{(i)}=\left(\kappa_{t_{\min }}^{(i+1)}, \ldots, \kappa_{t-1}^{(i+1)}, \kappa_{t+1}^{(i)}, \ldots, \kappa_{t_{\max }}^{(i)}\right)^{\prime}
$$


3. Afterwards generate a realization $u$ from the $\operatorname{Uniform}(0,1)$ distribution. If $u \leq$ $\psi\left(\kappa_{t}^{(i)}, \kappa_{t}^{*}\right)$ then the candidate is kept and $\kappa_{t}^{(i+1)}=\kappa_{t}^{*}$. On the contrary, if $u>\psi\left(\kappa_{t}^{(i)}, \kappa_{t}^{*}\right)$ then the candidate is rejected and the Markov chain does not move $\left(\kappa_{t}^{(i+1)}=\kappa_{t}^{(i)}\right)$.

4. Finally we have to transform

$$
\boldsymbol{\kappa}^{(i+1)}=\left(\kappa_{t_{\min }}^{(i+1)}, \ldots, \kappa_{t}^{(i+1)}, \kappa_{t+1}^{(i)}, \ldots, \kappa_{t_{\max }}^{(i)}\right)^{\prime}
$$

and $\boldsymbol{\alpha}^{(i)}$ in order to fulfill the constraints (1.3). To this end, we use the following formulas:

$$
\begin{aligned}
\boldsymbol{\kappa}^{(i+1)} & \leftarrow \boldsymbol{\kappa}^{(i+1)}-\overline{\boldsymbol{\kappa}} \\
\boldsymbol{\alpha}^{(i)} & \leftarrow \boldsymbol{\alpha}^{(i)}+\boldsymbol{\beta}^{(i)} \overline{\boldsymbol{\kappa}}
\end{aligned}
$$

where

$$
\overline{\boldsymbol{\kappa}}=\frac{1}{T}\left(\sum_{s \leq t} \kappa_{s}^{(i+1)}+\sum_{s>t} \kappa_{s}^{(i)}\right)
$$

Remark that the choice of parameter $\sigma_{t}^{2}$ is free but not neglectable. It directly influences acceptance rate of the proposals: a large variance will reduce the chance for the candidate to be kept and for the chain to move to another state. In practice we want the acceptation probability to be in the interval [20\%,50\%]. Therefore, a trial and error method is used to select $\sigma_{t}^{2}$. Starting from some initial value, we compute the acceptation probability (on about one hundred iterations, say). If it is too small, we have to increase the variance $\sigma_{t}^{2}$ (make it double, say). On the contrary, if more than half the candidates are kept, we reduce the value of $\sigma_{t}^{2}$.

\subsection{Metropolis-Hastings sampling for $\beta$}

The $\boldsymbol{\beta}$ update is quite similar to the $\boldsymbol{\kappa}$ one. Let us define

$$
\boldsymbol{\beta}_{-x}=\left(\beta_{x_{\min }}, \ldots, \beta_{x-1}, \beta_{x+1}, \ldots, \beta_{x_{\max }}\right)^{\prime}
$$

and in the same way

$$
\boldsymbol{D}_{-x}=\left(\boldsymbol{D}_{x_{\min }}, \ldots, \boldsymbol{D}_{x-1}, \boldsymbol{D}_{x+1}, \ldots, \boldsymbol{D}_{x_{\max }}\right)^{\prime}
$$


where $\boldsymbol{D}_{x}$ is the $\left(x-x_{\text {min }}+1\right)$-th row of $\boldsymbol{D}$. With the same developments as in previous section we find

$$
\begin{aligned}
& f\left(\beta_{x} \mid \boldsymbol{\beta}_{-x}, \boldsymbol{\alpha}, \boldsymbol{\kappa}, \boldsymbol{D}, \sigma_{\kappa}^{2}, \sigma_{\beta}^{2}, \boldsymbol{\gamma}, \rho\right) \\
& \propto \prod_{y=x_{\min }}^{x_{\max }} f\left(\beta_{y}, \boldsymbol{D}_{y} \mid \beta_{x_{\min }}, \ldots, \beta_{y-1}, \boldsymbol{D}_{x_{\min }}, \ldots, \boldsymbol{D}_{y-1}, \boldsymbol{\alpha}, \boldsymbol{\kappa}, \sigma_{\kappa}^{2}, \sigma_{\beta}^{2}, \boldsymbol{\gamma}, \rho\right) \\
& \propto \prod_{y=x_{\min }}^{x_{\max }} f\left(\boldsymbol{D}_{y} \mid \beta_{x_{\min }}, \ldots, \beta_{y-1}, \beta_{y}, \boldsymbol{D}_{x_{\min }}, \ldots, \boldsymbol{D}_{y-1}, \boldsymbol{\alpha}, \boldsymbol{\kappa}, \sigma_{\kappa}^{2}, \sigma_{\beta}^{2}, \boldsymbol{\gamma}, \rho\right) \\
& \prod_{y=x_{\min }}^{x_{\max }} f\left(\beta_{y} \mid \beta_{x_{\min }}, \ldots, \beta_{y-1}, \boldsymbol{D}_{x_{\min }}, \ldots, \boldsymbol{D}_{y-1}, \boldsymbol{\alpha}, \boldsymbol{\kappa}, \sigma_{\kappa}^{2}, \sigma_{\beta}^{2}, \boldsymbol{\gamma}, \rho\right) \\
& \propto \prod_{y=x_{\min }}^{x_{\max }} f\left(\boldsymbol{D}_{y} \mid \beta_{y}, \boldsymbol{\alpha}, \boldsymbol{\kappa}\right) f\left(\beta_{y}\right) \\
& \propto f\left(\boldsymbol{D}_{x} \mid \beta_{x}, \boldsymbol{\alpha}, \boldsymbol{\kappa}\right) f\left(\beta_{x}\right) \\
& \propto \prod_{t} \exp \left(-E_{x t} \exp \left(\alpha_{x}+\beta_{x} \kappa_{t}\right)\right) \prod_{t} \exp \left(\beta_{x} \kappa_{t} D_{x t}\right) \exp \left(-\frac{1}{2 \sigma_{\beta}^{2}} \beta_{x}^{2}\right) \text {. }
\end{aligned}
$$

Now we can decompose iteration $(i+1)$ of Metropolis-Hastings sampling for $\beta_{x}$ update. Suppose the parameter is estimated at iteration $i$ by $\beta_{x}^{(i)}$ and we have estimations $\beta_{y}^{(i+1)}$ for $y<x$. We then proceed as follows:

1. Select a candidate $\beta_{x}^{*}$ from the $\mathcal{N} \operatorname{ormal}\left(\beta_{x}^{(i)}, \sigma_{x}^{2}\right)$ distribution with known variance $\sigma_{x}^{2}$.

2. Compute the acceptance probability

$$
\psi\left(\beta_{x}^{(i)}, \beta_{x}^{*}\right)=\min \left(1, \frac{f\left(\beta_{x}^{*} \mid \boldsymbol{\beta}_{-x}^{(i)}, \boldsymbol{D}, \boldsymbol{\alpha}, \boldsymbol{\kappa}, \sigma_{\kappa}^{2}, \sigma_{\beta}^{2}, \boldsymbol{\gamma}, \rho\right)}{f\left(\beta_{x}^{(i)} \mid \boldsymbol{\beta}_{-x}^{(i)}, \boldsymbol{D}, \boldsymbol{\alpha}, \boldsymbol{\kappa}, \sigma_{\kappa}^{2}, \sigma_{\beta}^{2}, \boldsymbol{\gamma}, \rho\right)}\right)
$$

where

$$
\boldsymbol{\beta}_{-x}^{(i)}=\left(\beta_{x_{\min }}^{(i+1)}, \ldots, \beta_{x-1}^{(i+1)}, \beta_{x+1}^{(i)}, \ldots, \beta_{x_{\max }}^{(i)}\right)^{\prime}
$$

3. Afterwards generate a realization $u$ from the $\operatorname{Uniform}(0,1)$ distribution. If $u \leq$ $\psi\left(\beta_{x}^{(i)}, \beta_{x}^{*}\right)$, the candidate is kept and $\beta_{x}^{(i+1)}=\beta_{x}^{*}$. On the contrary, if $u>\psi\left(\beta_{x}^{(i)}, \beta_{x}^{*}\right)$, the candidate is rejected and the Markov chain does not move $\left(\beta_{x}^{(i+1)}=\beta_{x}^{(i)}\right)$.

4. Finally we have to transform vectors

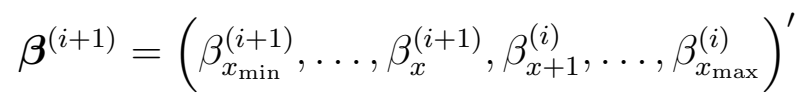

and $\boldsymbol{\kappa}^{(i+1)}$ in order to fulfill constraints (1.3):

$$
\begin{aligned}
& \boldsymbol{\beta}^{(i+1)} \leftarrow \frac{\boldsymbol{\beta}^{(i+1)}}{\boldsymbol{\beta}_{\bullet}} \\
& \boldsymbol{\kappa}^{(i+1)} \leftarrow \boldsymbol{\kappa}^{(i+1)} \boldsymbol{\beta} \bullet
\end{aligned}
$$


where

$$
\boldsymbol{\beta}_{\bullet}=\sum_{y \leq x} \beta_{y}^{(i+1)}+\sum_{y>x} \beta_{y}^{(i)}
$$

As for $\boldsymbol{\kappa}$ update, the variance $\sigma_{x}^{2}$ must be adjusted in order to have acceptance probabilities between $20 \%$ and $50 \%$.

\subsection{Gibbs sampling for $\alpha$}

Another standard approach which produces a Markov chain whose stationary distribution is consistent with the posterior distribution (3.1) is based on a variant of the Metropolis algorithm called the Gibbs sampler. It will enable us to exploit conditional densities to obtain realizations from the posterior density. The Gibbs sampler requires that the unknown model parameters are first assigned arbitrary values. Then an iterative sampling process takes place. At each iteration, the Gibbs sampler visits each unknown parameter in turn, and generates a random value from its full conditional distribution, conditional upon current values of all other parameters and upon the data. Then, each iteration yields a sample realization of the complete set of unknown parameters in the model. The generated realizations converge in distribution to the joint posterior distribution of the unknwown parameters.

Let us consider the likelihood function (2.1) as function of the vector $\boldsymbol{e}$ only:

$$
\begin{aligned}
L(\boldsymbol{e}, \boldsymbol{\beta}, \boldsymbol{\kappa}) & \propto \prod_{x} \prod_{t} \exp \left(-E_{x t} \exp \left(\alpha_{x}+\beta_{x} \kappa_{t}\right)+D_{x t}\left(\alpha_{x}+\beta_{x} \kappa_{t}\right)\right) \\
& \propto \prod_{x} \exp \left(-c_{x} e_{x}\right) e_{x}^{D_{x}}
\end{aligned}
$$

with

$$
c_{x}=\sum_{t} E_{x t} \exp \left(\beta_{x} \kappa_{t}\right) \text { and } D_{x}=\sum_{t} D_{x t} .
$$

To draw random samples from the posterior density, we use the Gibbs sampling algorithm. The essence of the Gibbs sampler lies in breaking a complicated joint probability density into a set of full conditional densities, and sampling one variable at a time, conditional on the values of the others.

For $x=x_{\min }, \ldots, x_{\max }$, we can write

$$
\begin{aligned}
f\left(e_{x} \mid \boldsymbol{\beta}, \boldsymbol{\kappa}, \boldsymbol{D}, \sigma_{\kappa}^{2}, \sigma_{\beta}^{2}, \boldsymbol{\gamma}, \rho\right) & =f\left(e_{x} \mid \boldsymbol{\beta}, \boldsymbol{\kappa}, \boldsymbol{D}\right) \\
& \propto f\left(e_{x}, \boldsymbol{\beta}, \boldsymbol{\kappa}, \boldsymbol{D}\right) f\left(e_{x}\right) \\
& \propto \exp \left(-c_{x} e_{x}\right) e_{x}^{D_{x} \bullet} e_{x}^{a_{x}-1} \exp \left(-b_{x} e_{x}\right) \\
& \propto \exp \left(-\left(b_{x}+c_{x}\right) e_{x}\right) e_{x}^{a_{x}+D_{x} \bullet-1}
\end{aligned}
$$

so that the distribution of $e_{x}$ given $\boldsymbol{\beta}, \boldsymbol{\kappa}, \boldsymbol{D}, \sigma_{\kappa}^{2}, \sigma_{\beta}^{2}, \boldsymbol{\gamma}, \rho$ is still gamma with updated parameters, that is,

$$
\left(e_{x} \mid \boldsymbol{\beta}, \boldsymbol{\kappa}, \boldsymbol{D}, \sigma_{\kappa}^{2}, \sigma_{\beta}^{2}, \boldsymbol{\gamma}, \rho\right) \sim \mathcal{G} a m m a\left(a_{x}+D_{x}, b_{x}+c_{x}\right) .
$$

Realizations of $e_{x}$ given $\boldsymbol{\beta}, \boldsymbol{\kappa}, \boldsymbol{D}, \sigma_{\kappa}^{2}, \sigma_{\beta}^{2}, \boldsymbol{\gamma}, \rho$ are thus easily generated. 


\subsection{Gibbs sampling for $\rho$}

From conditional distribution definition we have

$$
\begin{aligned}
f\left(\rho \mid \boldsymbol{\alpha}, \boldsymbol{\beta}, \boldsymbol{\kappa}, \boldsymbol{D}, \sigma_{\kappa}^{2}, \sigma_{\beta}^{2}, \boldsymbol{\gamma}\right) & =\frac{f\left(\rho, \boldsymbol{\alpha}, \boldsymbol{\beta}, \boldsymbol{\kappa}, \boldsymbol{D}, \sigma_{\kappa}^{2}, \sigma_{\beta}^{2}, \boldsymbol{\gamma}\right)}{f\left(\boldsymbol{\alpha}, \boldsymbol{\beta}, \boldsymbol{\kappa}, \boldsymbol{D}, \sigma_{\kappa}^{2}, \sigma_{\beta}^{2}, \boldsymbol{\gamma}\right)} \\
& \propto f\left(\boldsymbol{D} \mid \rho, \boldsymbol{\alpha}, \boldsymbol{\beta}, \boldsymbol{\kappa}, \sigma_{\kappa}^{2}, \sigma_{\beta}^{2}, \boldsymbol{\gamma}\right) f\left(\rho, \boldsymbol{\alpha}, \boldsymbol{\beta}, \boldsymbol{\kappa}, \sigma_{\kappa}^{2}, \sigma_{\beta}^{2}, \boldsymbol{\gamma}\right) \\
& =f(\boldsymbol{D} \mid \boldsymbol{\alpha}, \boldsymbol{\beta}, \boldsymbol{\kappa}) f\left(\boldsymbol{\kappa}, \rho \mid \boldsymbol{\alpha}, \boldsymbol{\beta}, \sigma_{\kappa}^{2}, \sigma_{\beta}^{2}, \boldsymbol{\gamma}\right) f\left(\boldsymbol{\alpha}, \boldsymbol{\beta}, \sigma_{\kappa}^{2}, \sigma_{\beta}^{2}, \boldsymbol{\gamma}\right) \\
& \propto f(\boldsymbol{\kappa}, \rho) \\
& =f(\boldsymbol{\kappa} \mid \rho) f(\rho) .
\end{aligned}
$$

The conditional density of $\boldsymbol{\kappa}$ given $\rho$ is given by

$$
f(\boldsymbol{\kappa} \mid \rho)=\prod_{t} f\left(\kappa_{t} \mid \kappa_{t-1}, \rho\right) \propto \exp \left(-\frac{1}{2 \sigma_{\kappa}^{2}}\left(a_{\rho} \rho^{2}-2 b_{\rho} \rho\right)\right)
$$

with

$$
a_{\rho}=\sum_{t}\left(\kappa_{t-1}-\eta_{t-1}\right)^{2} \text { and } b_{\rho}=\sum_{t}\left(\kappa_{t}-\eta_{t}\right)\left(\kappa_{t-1}-\eta_{t-1}\right)
$$

and the convention $\kappa_{t_{\min }-1}=\eta_{t_{\min }-1}$. Therefore,

$$
\begin{aligned}
f\left(\rho \mid \boldsymbol{\alpha}, \boldsymbol{\beta}, \boldsymbol{\kappa}, \boldsymbol{D}, \sigma_{\kappa}^{2}, \sigma_{\beta}^{2}, \boldsymbol{\gamma}\right) & \propto \exp \left(-\frac{1}{2 \sigma_{\kappa}^{2}}\left(a_{\rho} \rho^{2}-2 b_{\rho} \rho\right)\right) \exp \left(-\frac{1}{2 \sigma_{\rho}^{2}} \rho^{2}\right) \\
& \propto \exp \left(-\frac{1}{2 \sigma_{\rho}^{2^{*}}}\left(\rho-\mu_{\rho}^{*}\right)^{2}\right)
\end{aligned}
$$

with

$$
\mu_{\rho}^{*}=\frac{b_{\rho}}{a_{\rho}+\frac{\sigma_{\kappa}^{2}}{\sigma_{\rho}^{2}}} \text { and } \sigma_{\rho}^{2^{*}}=\frac{\sigma_{\kappa}^{2}}{a_{\rho}+\frac{\sigma_{\kappa}^{2}}{\sigma_{\rho}^{2}}} .
$$

The distribution of $\rho$ given $\boldsymbol{\alpha}, \boldsymbol{\beta}, \boldsymbol{\kappa}, \boldsymbol{D}, \sigma_{\kappa}^{2}, \sigma_{\beta}^{2}, \boldsymbol{\gamma}$ can then be written as

$$
\left(\rho \mid \boldsymbol{\alpha}, \boldsymbol{\beta}, \boldsymbol{\kappa}, \boldsymbol{D}, \sigma_{\kappa}^{2}, \sigma_{\beta}^{2}, \gamma\right) \sim \mathcal{N} \operatorname{ormal}\left(\mu_{\rho}^{*}, \sigma_{\rho}^{2^{*}}\right) \text { truncated to }(-1,1) .
$$

Simulation from (3.7) is easy.

\subsection{Gibbs sampling for $\sigma_{\kappa}^{2}$}

By using the same principles as previously we find

$$
f\left(\sigma_{\kappa}^{2} \mid \boldsymbol{\alpha}, \boldsymbol{\beta}, \boldsymbol{\kappa}, \boldsymbol{D}, \sigma_{\beta}^{2}, \boldsymbol{\gamma}, \rho\right) \propto f\left(\boldsymbol{\kappa} \mid \sigma_{\kappa}^{2}\right) f\left(\sigma_{\kappa}^{2}\right) .
$$

Since

$$
f\left(\boldsymbol{\kappa} \mid \sigma_{\kappa}^{2}\right)=\frac{1}{2 \pi \sigma_{\kappa}^{2}} \exp \left(-\frac{1}{2 \sigma_{\kappa}^{2}} \sum_{t}\left(\kappa_{t}-\eta_{t}-\rho\left(\kappa_{t-1}-\eta_{t-1}\right)\right)^{2}\right)
$$


and the prior distribution of $\sigma_{\kappa}^{-2}$ is $\mathcal{G} a m m a\left(a_{\kappa}, b_{\kappa}\right)$, we find $f\left(\sigma_{\kappa}^{2} \mid \boldsymbol{\alpha}, \boldsymbol{\beta}, \boldsymbol{\kappa}, \boldsymbol{D}, \sigma_{\beta}^{2}, \boldsymbol{\gamma}, \rho\right) \propto \sigma_{\kappa}{ }^{-2\left(a_{\kappa}+1+\frac{T}{2}\right)} \exp \left(-\frac{1}{\sigma_{\kappa}^{2}}\left(b_{\kappa}+\frac{1}{2} \sum_{t}\left(\kappa_{t}-\eta_{t}-\rho\left(\kappa_{t-1}-\eta_{t-1}\right)\right)^{2}\right)\right)$

with $\kappa_{t_{\min }-1}=\eta_{t_{\min }-1}$. The distribution of $\sigma_{\kappa}^{-2}$ given $\boldsymbol{\alpha}, \boldsymbol{\beta}, \boldsymbol{\kappa}, \boldsymbol{D}, \sigma_{\beta}^{2}, \boldsymbol{\gamma}, \rho$ is then given by

$$
\left(\sigma_{\kappa}^{-2} \mid \boldsymbol{\alpha}, \boldsymbol{\beta}, \boldsymbol{\kappa}, \boldsymbol{D}, \sigma_{\beta}^{2}, \boldsymbol{\gamma}, \rho\right) \sim \mathcal{G} a m m a\left(a_{\kappa}+\frac{T}{2}, b_{\kappa}+\frac{1}{2} \sum_{t}\left(\kappa_{t}-\eta_{t}-\rho\left(\kappa_{t-1}-\eta_{t-1}\right)\right)^{2}\right) .
$$

\subsection{Gibbs sampling for $\sigma_{\beta}^{2}$}

Applying the same reasoning as before, we find

$$
\begin{aligned}
f\left(\sigma_{\beta}^{2} \mid \boldsymbol{\alpha}, \boldsymbol{\beta}, \boldsymbol{\kappa}, \boldsymbol{D}, \sigma_{\kappa}^{2}, \boldsymbol{\gamma}, \rho\right) & \propto f\left(\boldsymbol{\beta} \mid \sigma_{\beta}^{2}\right) f\left(\sigma_{\beta}^{2}\right) \\
& \propto \sigma_{\beta}{ }^{-2\left(a_{\beta}+1+\frac{M}{2}\right)} \exp \left(-\frac{1}{\sigma_{\beta}^{2}}\left(b_{\beta}+\frac{1}{2} \boldsymbol{\beta}^{\prime} \boldsymbol{\beta}\right)\right) .
\end{aligned}
$$

The distribution of $\sigma_{\beta}^{-2}$ given $\boldsymbol{\alpha}, \boldsymbol{\beta}, \boldsymbol{\kappa}, \boldsymbol{D}, \sigma_{\kappa}^{2}, \boldsymbol{\gamma}, \rho$ is then given by

$$
\left(\sigma_{\beta}^{-2} \mid \boldsymbol{\alpha}, \boldsymbol{\beta}, \boldsymbol{\kappa}, \boldsymbol{D}, \sigma_{\kappa}^{2}, \boldsymbol{\gamma}, \rho\right) \sim \mathcal{G} a m m a\left(a_{\beta}+\frac{M}{2}, b_{\beta}+\frac{1}{2} \boldsymbol{\beta}^{\prime} \boldsymbol{\beta}\right)
$$

\subsection{Gibbs sampling for $\gamma$}

As previously we have

$$
f\left(\boldsymbol{\gamma} \mid \boldsymbol{\kappa}, \sigma_{\kappa}^{2}, \sigma_{\beta}^{2}, \rho\right) \propto f\left(\boldsymbol{\kappa} \mid \boldsymbol{\gamma}, \rho, \sigma_{\kappa}^{2}\right) f(\boldsymbol{\gamma})
$$

Because $\boldsymbol{\gamma}$ is a priori distributed according to $\mathcal{N}$ ormal $_{2}\left(\boldsymbol{\gamma}_{0}, \boldsymbol{\Sigma}_{0}\right)$, we find

$$
\begin{aligned}
f\left(\boldsymbol{\gamma} \mid \boldsymbol{\kappa}, \sigma_{\kappa}^{2}, \sigma_{\beta}^{2}, \rho\right) & \propto \exp \left(-\frac{1}{2 \sigma_{\kappa}^{2}}(\boldsymbol{\kappa}-\boldsymbol{X} \boldsymbol{\gamma})^{\prime} \boldsymbol{Q}(\boldsymbol{\kappa}-\boldsymbol{X} \boldsymbol{\gamma})\right) \exp \left(-\frac{1}{2}\left(\boldsymbol{\gamma}-\boldsymbol{\gamma}_{0}\right)^{\prime} \boldsymbol{\Sigma}_{0}^{-1}\left(\boldsymbol{\gamma}-\boldsymbol{\gamma}_{0}\right)\right) \\
& \propto \exp \left(-\frac{1}{2 \sigma_{\kappa}^{2}}\left(\boldsymbol{\gamma}-\boldsymbol{\gamma}^{*}\right)^{\prime} \boldsymbol{\Sigma}^{*-1}\left(\boldsymbol{\gamma}-\boldsymbol{\gamma}^{*}\right)\right)
\end{aligned}
$$

with

$$
\boldsymbol{\Sigma}^{*}=\left(\boldsymbol{X}^{\prime} \boldsymbol{Q} \boldsymbol{X}+\sigma_{\kappa}^{2} \boldsymbol{\Sigma}_{0}^{-1}\right)^{-1} \text { and } \boldsymbol{\gamma}^{*}=\boldsymbol{\Sigma}^{*}\left(\boldsymbol{X}^{\prime} \boldsymbol{Q} \boldsymbol{\kappa}+\sigma_{\kappa}^{2} \boldsymbol{\Sigma}_{0}^{-1} \boldsymbol{\gamma}_{0}\right)
$$

Thus the distribution of $\boldsymbol{\gamma}$ given $\boldsymbol{\kappa}, \sigma_{\kappa}^{2}, \sigma_{\beta}^{2}, \rho$ is

$$
\left(\gamma \mid \boldsymbol{\kappa}, \sigma_{\kappa}^{2}, \sigma_{\beta}^{2}, \rho\right) \sim \mathcal{N}_{\operatorname{ormal}_{2}}\left(\boldsymbol{\gamma}^{*}, \sigma_{\kappa}^{2} \boldsymbol{\Sigma}^{*}\right)
$$




\section{Numerical illustration}

\subsection{Data set}

Our data are about French male population aged 0 to 89 between 1950 and 2000. The data related to calendar years 1950 to 1997 come from INED (Institut National d'Etudes Démographiques based in Paris, France). Those related to calendar years 1998, 1999 and 2000 have been obtained from INSEE (Institut National de la Statistique et des Etudes Economiques based in Paris, France). The following information is available: the numbers $L_{x t}$ of people aged $x$ on January 1 of year $t$ (for $x$ between 0 and 89), and the numbers $D_{x t}$ of people aged $x$ dying during year $t$. The exposure-to-risk $E_{x t}$ is then computed under assumption (1.1).

\subsection{Initialization and choice of prior distributions for the hyper- parameters}

As seen in the previous section, we work with three vectors of parameters $(\boldsymbol{\alpha}, \boldsymbol{\beta}$ and $\boldsymbol{\kappa})$ and with five hyperparameters $\left(\rho, \sigma_{\kappa}^{2}, \sigma_{\beta}^{2}\right.$ and the two components of $\left.\gamma\right)$. We also have to fix the constants $a_{\kappa}, b_{\kappa}, a_{\beta}, b_{\beta}, \sigma_{\rho}^{2}$, the $M$ components $a_{x}$, the $M$ components $b_{x}$, the vector $\gamma_{0}$ and the matrix $\Sigma_{0}$ involved in the distribution of the hyperparameters.

The choices for the constants determining the distributions of the hyperparameters are made in an empirical Bayes approach. In empirical Bayes, hyperparameters from the last level of a hierarchical model are estimated rather than chosen a priori. Although this procedure might seem better because it lets the data decide about reasonable values for obscure hyperparameters, many theoretical arguments have been levelled against it. Despite the inferential problems, this procedure is often used (namely because using the data in this way turns out to be equivalent to making the prior indifferent to certain chosen parameters; see e.g. Carlin \& Louis (2000)).

Specifically, we first compute the frequentist estimates of $\boldsymbol{\alpha}, \boldsymbol{\beta}$ and $\boldsymbol{\kappa}$ by maximizing the likelihood (2.1), as described in Brounns ET AL. (2002a,b). Because of the presence of the bilinear term $\beta_{x} \kappa_{t}$, it is not possible to estimate the proposed model with commercial statistical packages that implement Poisson regression. A uni-dimensional or elementary Newton method is used instead as proposed by Goodman (1979) for estimating log-linear models with bilinear terms. The estimators of $\boldsymbol{\alpha}, \boldsymbol{\beta}$ and $\boldsymbol{\kappa}$ obtained in this way are further referred to as Goodman estimates.

We take for $\gamma_{0}$ the estimated parameters of a linear regression of $\boldsymbol{\kappa}$ on calendar time. Then, $\boldsymbol{\Sigma}_{0}$ is taken to be the estimated covariance matrix of these estimates. Afterwards, $\rho$ and $\sigma_{\kappa}^{2}$ can be initialized by fitting an $\operatorname{AR}(1)$ model on $\left(\boldsymbol{\kappa}-\boldsymbol{\gamma}_{0} X\right)$. Finally $\sigma_{\beta}^{2}$ is initialized to be the empirical variance of the Goodman $\widehat{\beta}_{x}$ 's.

When the model (1.2) is fitted by ordinary least-squares, the fitted values of $\alpha_{x}$ exactly equal the average of $\ln \widehat{\mu_{x}}(t)$ over time $t$ so that $\exp \alpha_{x}$ is the general shape of the mortality schedule. Also, in the Poisson $\log$-bilinear model, the fitted $\exp \alpha_{x}$ mimick the observed average of the $\widehat{\mu_{x}}(t)$ 's. In order to obtain an uninformative prior distribution (i.e. with large variance), we have to choose a small $b_{x}, 0.001$ say. Afterwards, $a_{x}$ can be choosen equal to $b_{x} \exp \widehat{\alpha}_{x}\left(\right.$ so that $\left.\mathbb{E}\left[e_{x}\right]=\exp \widehat{\alpha}_{x}\right)$. 
In the same way, if $\sigma_{\beta}^{-2} \sim \mathcal{G} a m m a\left(a_{\beta}, b_{\beta}\right)$, then $\mathbb{E}\left[\sigma_{\beta}^{2}\right]=\frac{b_{\beta}}{a_{\beta}-1}$ for $a_{\beta}>1$ and $\operatorname{Var}\left[\sigma_{\beta}^{2}\right]=$ $\frac{b_{\beta}^{2}}{\left(a_{\beta}-1\right)^{2}\left(a_{\beta}-2\right)}$ for $a_{\beta}>2$. So constants $a_{\beta}$ and $b_{\beta}$ control prior mean and variance of $\sigma_{\beta}^{2}$. Taking $a_{\beta}$ near to (but greater than) 2 will give a huge variance. So we find $b_{\beta}=\left(a_{\beta}-1\right) \sigma_{\beta}^{2}$. The same argument can be used for $a_{\kappa}$ and $b_{\kappa}$. We use $a_{\beta}=a_{\kappa}=2.1$ for the application.

Finally the initial value of $\sigma_{\rho}^{2}$ does not seem to be very influential since $\rho$ is restricted to interval $(0,1)$. We choose $\sigma_{\rho}^{2}=1$.

It is worth to mention that other constants have also been used. Typically, we have increased the a priori variance of parameters and hyperparameters. These more diffuse prior choices were based on smaller values of $b_{x}$ (from 0.001 to 0.00001 ), values of $a_{\beta}$ closer to 2 (from 2.1 to 2.00001), and "greater" matrix $\boldsymbol{\Sigma}_{0}$ (up to 10 times the initial matrix). The results were similar to those obtained with the initial values deduced from the empirical Bayes approach.

Instead of basing the prior choices of the parameters on the Goodman's estimations of $\boldsymbol{\alpha}$, $\boldsymbol{\beta}$ and $\boldsymbol{\kappa}$, we also used the Lee-Carter estimates to fix the constants and init hyperparameters and parameters involved in the model (in an empirical Bayes setting). Again, the results obtained in this way were almost identical to those obtained with the Goodman's estimations.

\subsection{Convergence diagnostics}

In practice, we typically run the Gibbs sampler for an initial period of a few thousands cycles and then collect information from several further thousands of cycles (of which we store every 10th for the subsequent construction of approximate interval estimates). The posterior means are estimated by the corresponding sample means. Here, 20000 iterations are computed. The first 10000 iterations are considered as the burn-in period. The last 10 000 iterations are used for estimation of the posterior distribution.

A sample from the distribution of interest (the posterior distribution of the parameter in our example) is only attained with MCMC when the number of iterations of the chain approaches infinity. This is of course impossible in practice and a value obtained at a sufficiently large iteration is taken instead. This raises the question of how large this iteration should be.

Some informal checks of convergence based on graphical techniques are commonly used. Several chains can be run in parallel with different initial states or a single chain is screened for exhibiting the same qualitative behavior through iterations (often after a transient initial period). More formal diagnostics are developed in Cowles \& CARLIN (1996) and Brooks \& RoBerts (1998).

Figure 4.1 gives the selected $\sigma_{t}^{2}$ and $\sigma_{x}^{2}$ and corresponding acceptation probabilities. These variances are selected by a trial an error method. Algorithm starts with $\sigma_{x}^{2}=\sigma_{t}^{2}=1$ for all $x$ and $t$, and a first 100-iteration-pilot run is computed. Variance $\sigma_{x}^{2}$ (or $\sigma_{t}^{2}$ ) is made double if the corresponding acceptation probability is below 20\%; it is divided by 2 if corresponding acceptation probability is greater than 50\%. A second 100-iteration-pilot run is then computed and variances $\sigma_{x}^{2}$ and $\sigma_{t}^{2}$ are moving as previously. The algorithm stops when rates for each age $x$ and each year $t$ are between $20 \%$ and $50 \%$. We observe larger variances $\sigma_{x}^{2}$ where mortality is very specific, i.e. between ages 0 and 40 (new borns, accidental hump). In the same way variance $\sigma_{t}^{2}$ grows with year $t$. 
Once we know how to sample from the a posteriori distribution of the parameters, we can compute a posteriori means by averaging the values obtained by repeating these steps a large number of times (after having discarded a suitable number of "burn-in" iterations to ensure that the algorithm has converged). Note that we do not worry about autocorrelation as long as we are not computing standard errors. Figure 4.2 shows the trajectory of the ergodic averages. Clearly, the asymptotic behavior is attained after the first 10000 runs.

In order to get samples with no significant autocorrelations, we take only one realization of each parameter by each 10-iteration-block after a burn-in period of 10000 iterations. We then have a sample of size $10000 / 10=1000$ from the a posteriori distribution of each parameter. Autocorrelations of these series are depicted in Figure 4.3. We clearly see there that no significant autocorrelations remain in the samples.

\subsection{Comparison with other methods}

Since the Lee-Carter method is the most popular one, it is interesting to compare MCMC estimates to this benchmark. To obtain the Lee-Carter estimates the model (1.2) is fitted to the matrix of age-specific observed forces of mortality using singular value decomposition (SVD). Then, $\widehat{\alpha_{x}}$ is taken to be the row average of the $\ln \widehat{\mu_{x}}(t)$ 's, and the $\widehat{\beta_{x}}$ 's and $\widehat{\kappa_{t}}$ 's are obtained from the first term of a SVD of the matrix $\ln \widehat{\mu_{x}}(t)-\widehat{\alpha_{x}}$.

Figures 4.4-4.5-4.6 depict estimates of $\alpha_{x}$ 's, $\beta_{x}$ 's and $\kappa_{t}$ 's according to the Lee-Carter SVD algorithm, the Poisson Goodman algorithm and the MCMC algorithm. Some numerical values for several ages $x$ and years $t$ can be viewed at Table 4.1. From Figure 4.4, we se that the pattern of the estimated $\alpha_{x}$ is almost identical whatever the estimation method used. On the contrary, Figures 4.5-4.6 show that there are significant differences in the $\widehat{\kappa}_{t}$ and $\widehat{\beta}_{x}$ patterns. The values obtained with Lee-Carter SVD lie outside the MCMC credible intervals for numerous ages and calendar years.

Table 4.2 displays the mortality rates for the generation aged 30 in calendar year 1950 . Whereas the values obtained with the Poisson log-bilinear model always lie inside the $95 \%$ MCMC credible interval, those derived from the Lee-Carter methodology are about half the time outside this interval. These results highlight significant differences with Lee-Carter estimates. They are nevertheless in agreement with those obtained with the Poisson logblinear model.

\section{Acknowledgements}

Antoine Delwarde was supported by a research grant from the Fonds de la Recherche pour l'Industrie et l'Agriculture - FRIA. This financial support is gratefully acknowledged.

\section{References}

Alho, J.M. (2000). Discussion of Lee (2000). North American Actuarial Journal 4, 91-93. BRILlinger, D.R. (1986). The natural variability of vital rates and associated statistics. Biometrics 42, 693-734. 

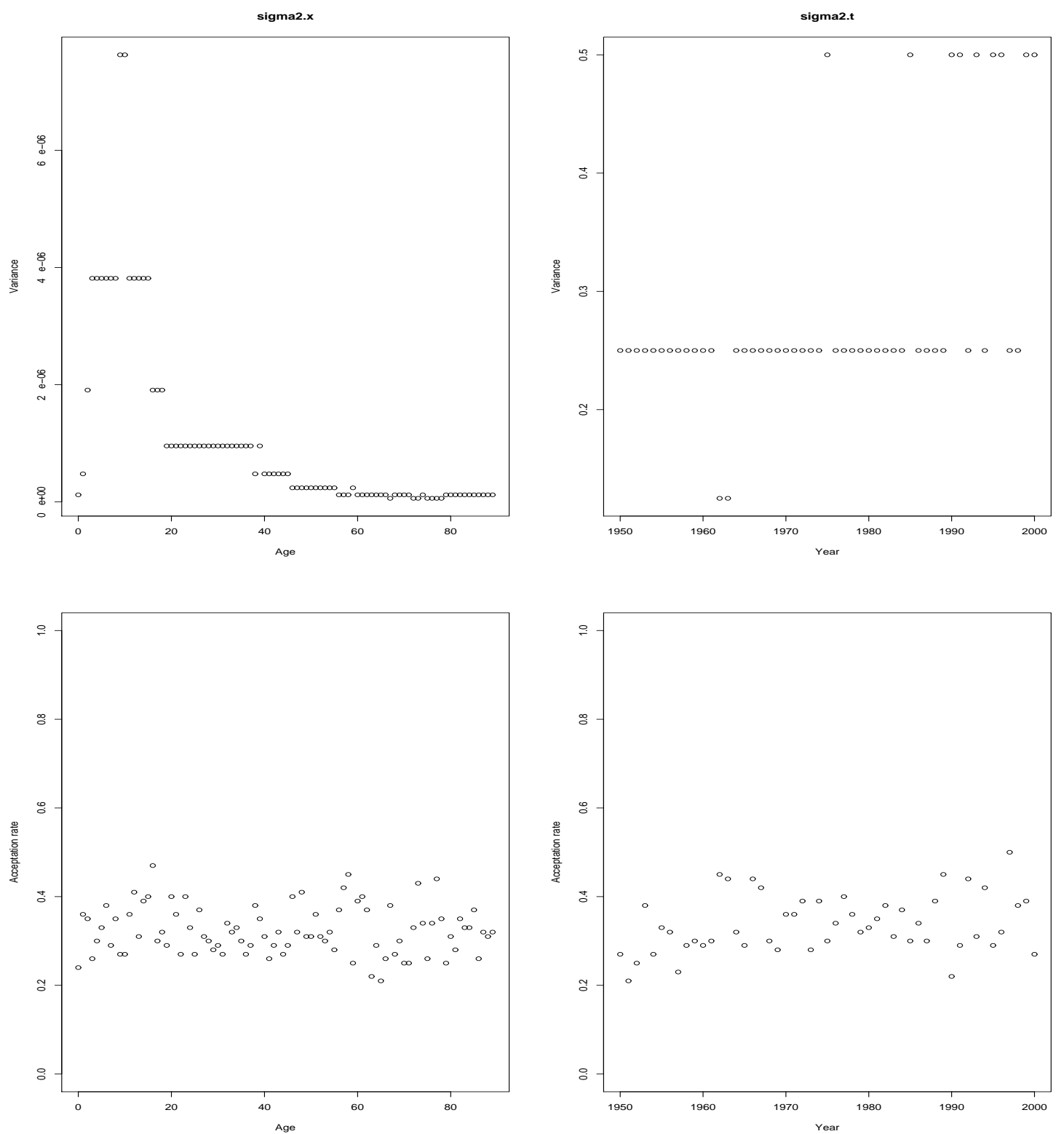

Figure 4.1: Choosen proposal variances (top panel with $\sigma_{x}^{2}$ to the left and $\sigma_{t}^{2}$ to the right) and corresponding acceptance rates after the last 100-iteration-pilot run (bottom panel). 

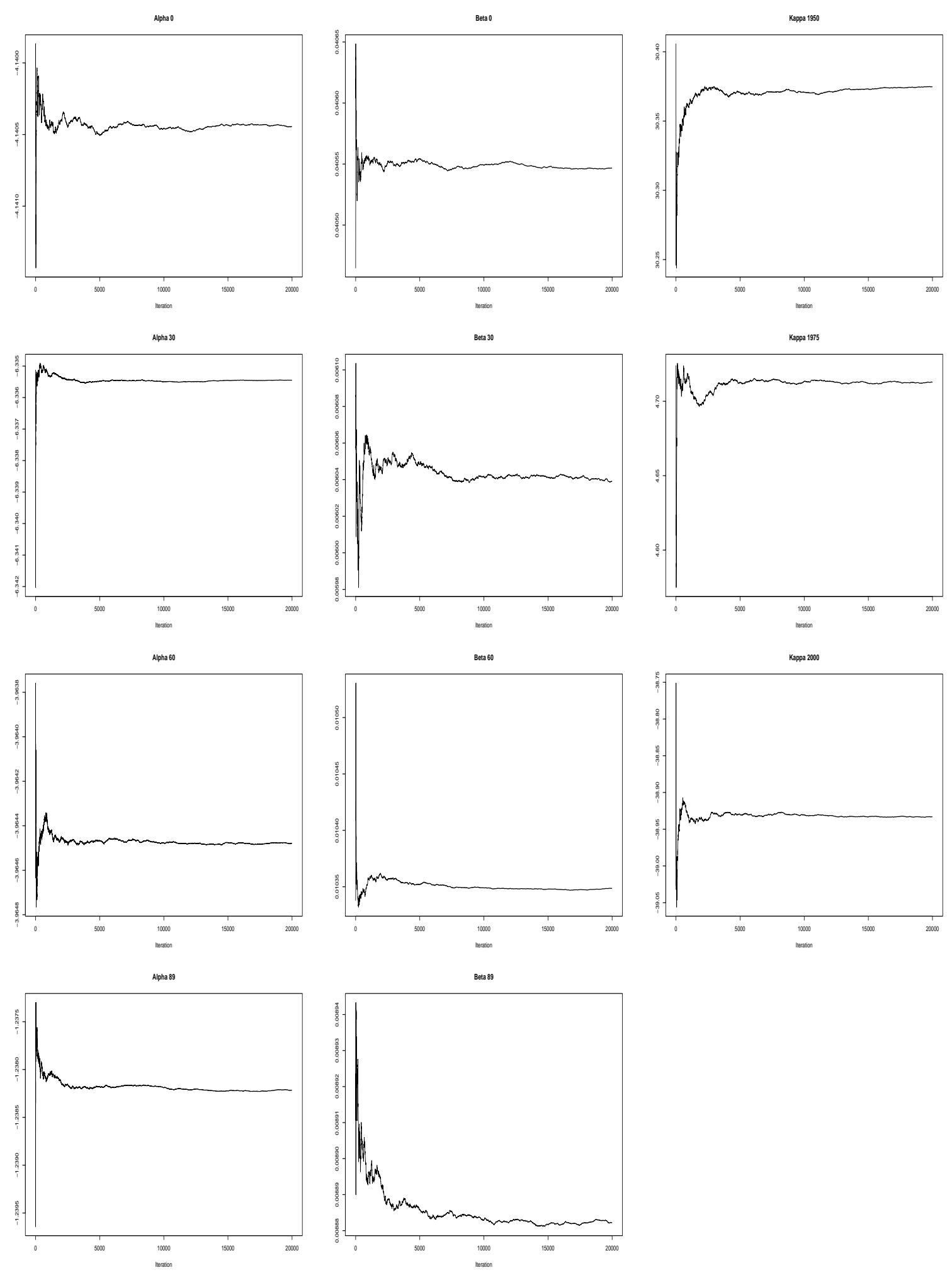

Figure 4.2: Ergodic mean of parameters $\alpha_{x}$ (left), $\beta_{x}$ (center) and $\kappa_{t}$ (right) for some selected ages $(0,30,60$ and 89) and years (1950, 1975 and 2000). 

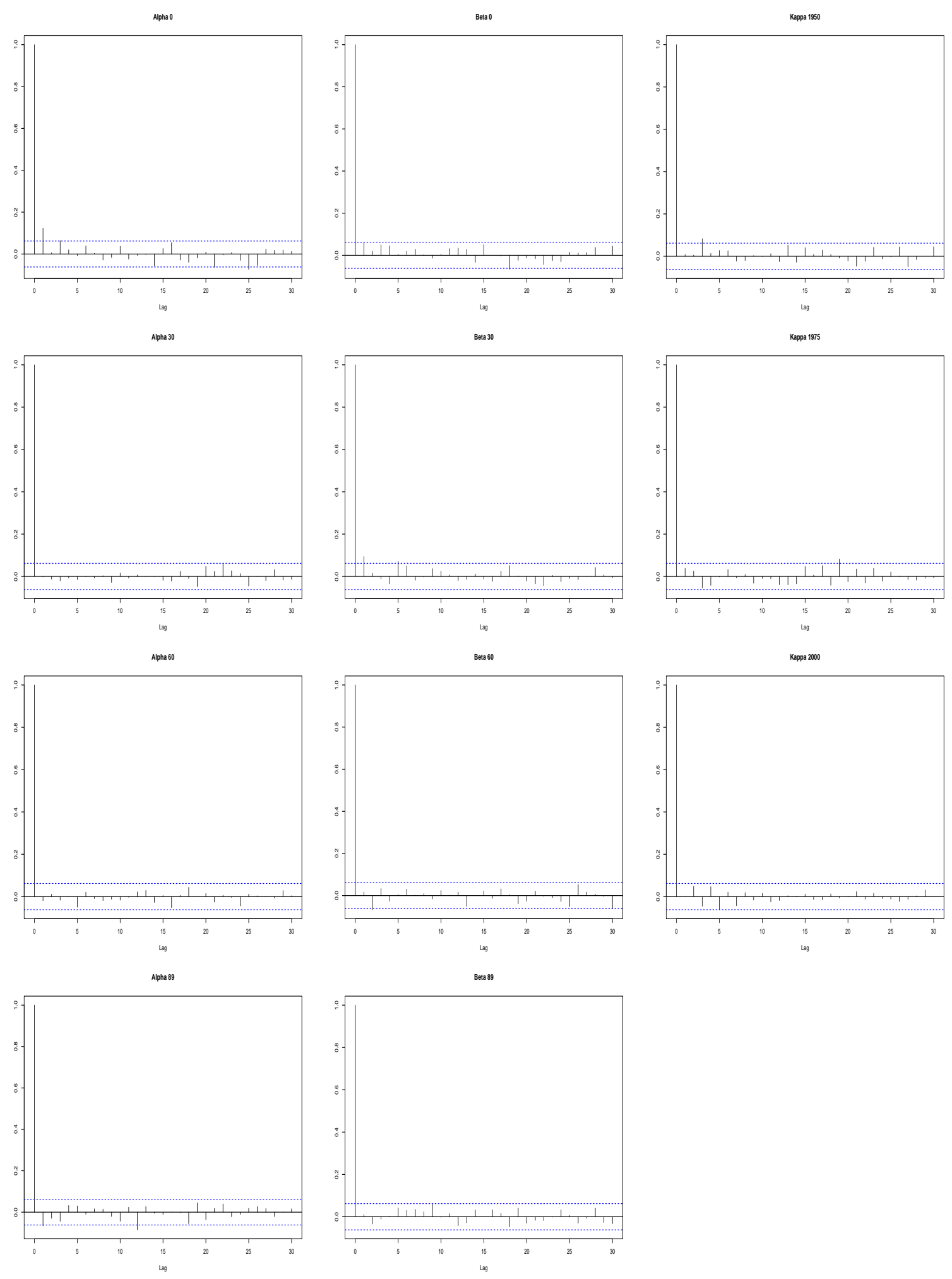

Figure 4.3: Autocorrelations of parameters $\alpha_{x}$ (left), $\beta_{x}$ (center) and $\kappa_{t}$ (right) for some selected ages (0, 30, 60 and 89) and years (1950, 1975 and 2000) based on 1000 recorded iterations after a burn-in period of 10000 iterations. 


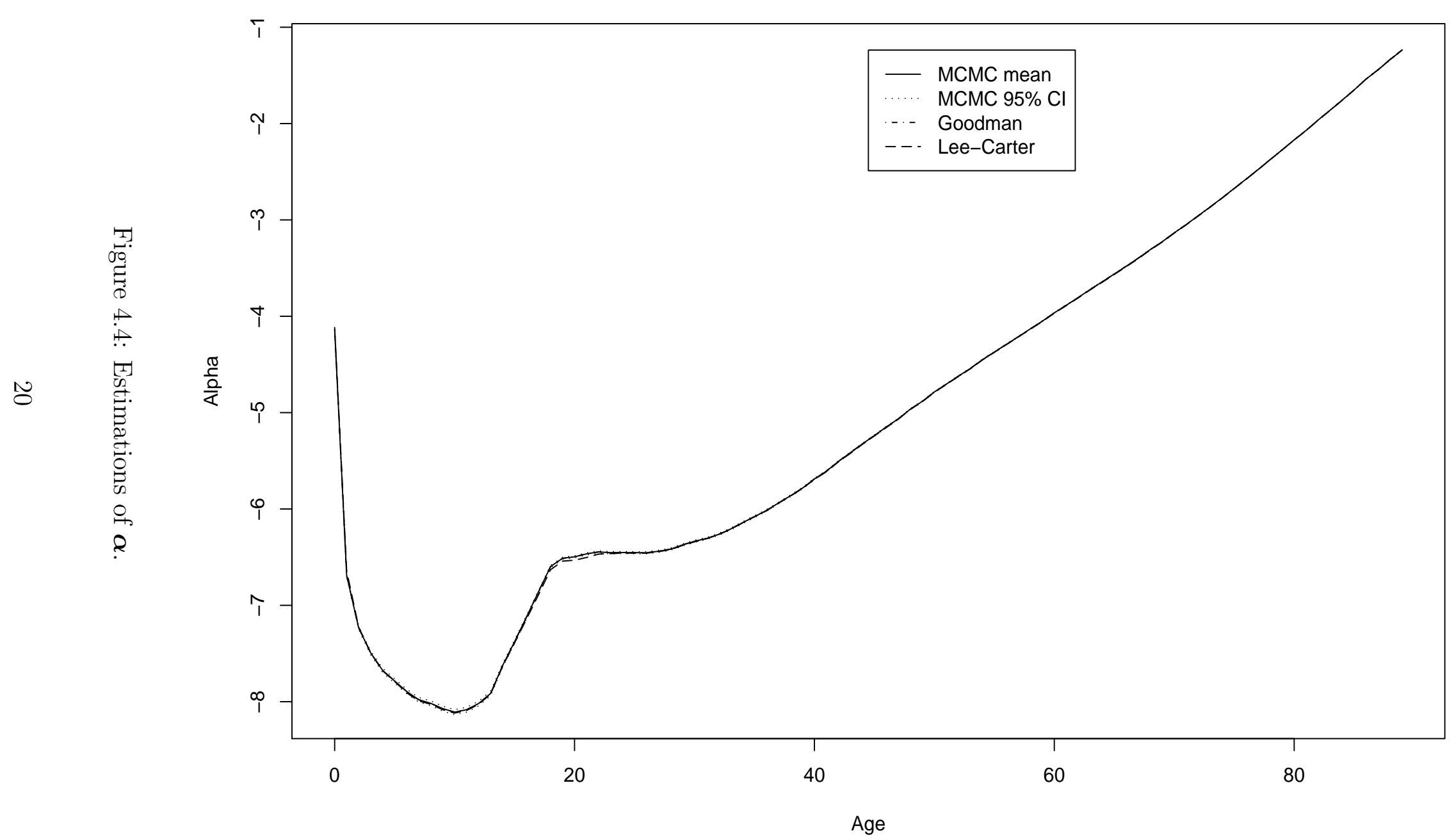




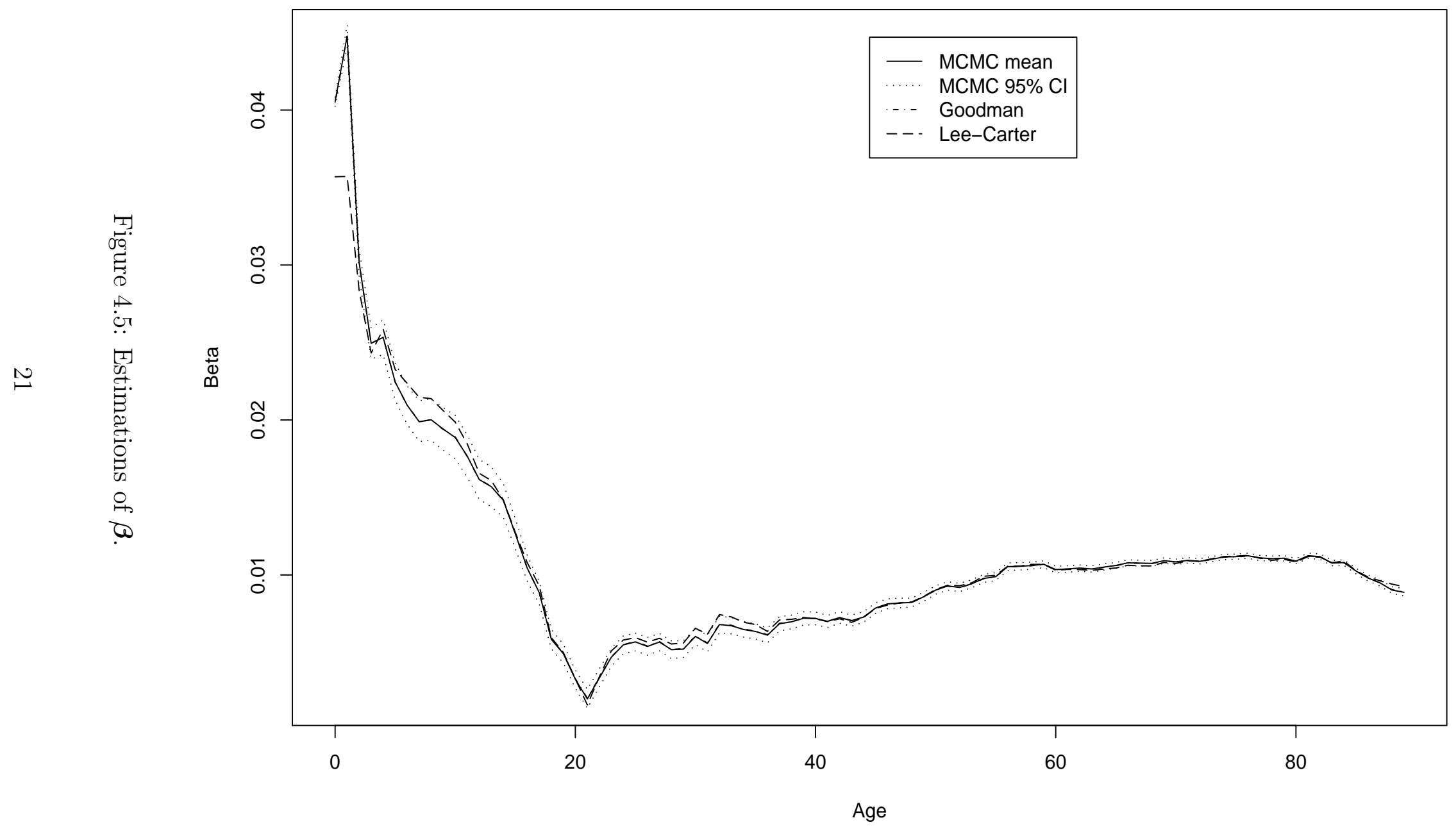




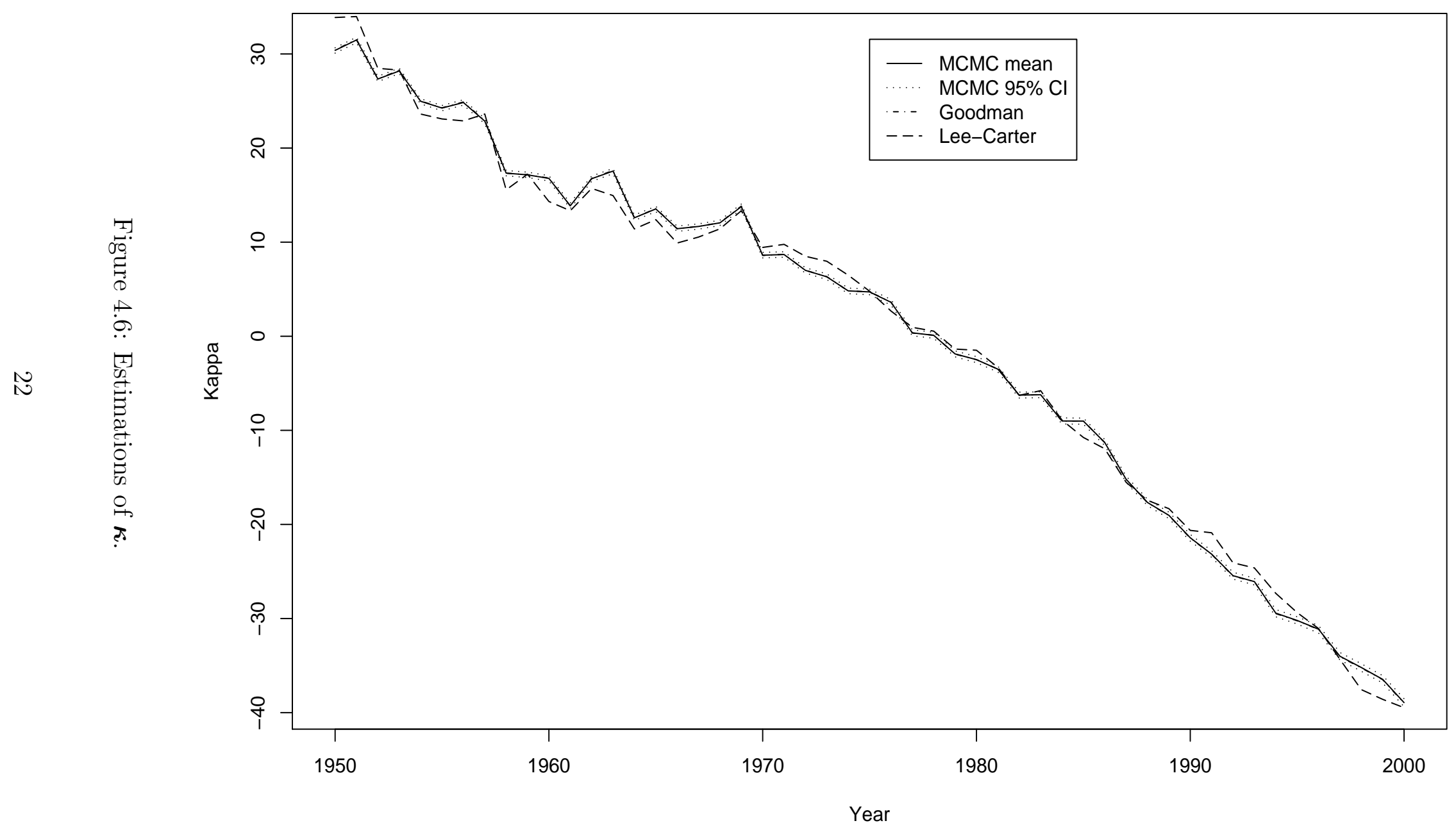




\begin{tabular}{|c|c|c|c|c|}
\hline \multirow[b]{2}{*}{$\alpha_{x}$} & \multicolumn{4}{|c|}{$x$} \\
\hline & 0 & 30 & 60 & 89 \\
\hline MCMC mean & -4.140164 & -6.335586 & -3.964490 & -1.238194 \\
\hline MCMC median & -4.140165 & -6.335663 & -3.964502 & -1.238182 \\
\hline $95 \%$ MCMC CI & {$[-4.144406,-4.136435]$} & {$[-6.346393,-6.324829]$} & {$[-3.968566,-3.960367]$} & {$[-1.243415,-1.233119]$} \\
\hline Goodman estimate & -4.140167 & -6.335124 & -3.964446 & -1.237482 \\
\hline Lee-Carter estimate & -4.115651 & -6.340877 & -3.968640 & -1.235153 \\
\hline
\end{tabular}

\begin{tabular}{|l|cccc|}
\hline & & & $x$ & \\
$\beta_{x}$ & 0 & 30 & 0.010351 & 89 \\
\hline MCMC mean & 0.040547 & 0.006034 & 0.010351 & 0.008887 \\
MCMC median & 0.040546 & 0.006039 & 0.008887 \\
95\% MCMC CI & {$[0.040255,0.040852]$} & {$[0.005479,0.006568]$} & {$[0.010125,0.010578]$} & {$[0.008646,0.009116]$} \\
Goodman estimate & 0.040465 & 0.006057 & 0.010338 & 0.008911 \\
Lee-Carter estimate & 0.035692 & 0.006561 & 0.010307 & 0.009243 \\
\hline
\end{tabular}

\begin{tabular}{|l|ccc|}
\hline & & $t$ & \\
$\kappa_{t}$ & 1950 & 1975 & 2000 \\
\hline MCMC mean & 30.383239 & 4.708426 & -38.939200 \\
MCMC median & 30.382987 & 4.707171 & -38.937704 \\
$95 \%$ MCMC CI & {$[30.096920,30.667779]$} & {$[4.418210,5.002447]$} & {$[-39.356280,-38.529110]$} \\
Goodman estimate & 30.405785 & 4.723949 & -38.998415 \\
Lee-Carter estimate & 33.875870 & 4.789799 & -39.475417 \\
\hline
\end{tabular}

Table 4.1: Estimations of $\alpha_{x}, \beta_{x}$ and $\kappa_{t}$ for several ages $x$ and years $t$.

Brooks, S.P. \& Roberts G.O. (1998). Assessing convergence of Markov Chain Monte Carlo algorithms. Statistics and Computing 8, 319-335.

Brouhns, N., Denuit, M. \& Vermunt, J.K. (2002a). A Poisson log-bilinear approach to the construction of projected lifetables. Insurance: Mathematics 85 Economics 31, 373-393.

Brouhns, N., Denuit, M. \& Vermunt, J.K. (2002b). Measuring the longevity risk in mortality projections. Bulletin of the Swiss Association of Actuaries, 105-130.

Carlin, B.P., \& Louis, T.A. (2000). Bayes and Empirical Bayes Methods for Data Analysis. Chapman and Hall/CRC.

Cowles, M.K. \& Carlin, B.P. (1996). Markov Chain Monte Carlo convergence diagnostics: A comparative review. Journal of the American Statistical Association 91, 883-904.

ChiB, S. (2001). Markov Chain Monte Carlo methods: Computation and inference. Handbook of Econometrics, Volume 5, 381-394. North Holland.

Gilks, W., Richardson, S. \& Spiegelhalter D. (1996). Markov Chain Monte Carlo in Practice. Chapman and Hall, New-York.

Girosi, F., \& King, G. (2003). Demographic Forecasting. Monograph.

Goodman, L.A. (1979). Simple models for the analysis of association in cross-classifications having ordered categories. Journal of the American Statistical Association 74, 537-552.

LEE, R.D. (2000). The Lee-Carter method of forecasting mortality, with various extensions and applications. North American Actuarial Journal 4, 80-93.

LeE, R.D., \& CARTER, L. (1992). Modelling and forecasting the time series of US mortality. Journal of the American Statistical Association 87, 659-671. 


\begin{tabular}{|c|c|c|c|c|c|c|}
\hline Age & Year & MCMC mean & MCMC median & MCMC 95\% CI & Goodman & Lee-Carter \\
\hline$\overline{30}$ & 1950 & 0.00213 & 0.00213 & {$[0.00209,0.00217]$} & 0.00213 & 0.00220 \\
\hline 31 & 1951 & 0.00217 & 0.00217 & {$[0.00213,0.00222]$} & 0.00218 & 0.00224 \\
\hline 32 & 1952 & 0.00229 & 0.00229 & {$[0.00225,0.00233]$} & 0.00229 & 0.00235 \\
\hline 33 & 1953 & 0.00243 & 0.00243 & {$[0.00238,0.00248]$} & 0.00244 & 0.00247 \\
\hline 34 & 1954 & 0.00252 & 0.00252 & {$[0.00248,0.00257]$} & 0.00253 & 0.00253 \\
\hline 35 & 1955 & 0.00267 & 0.00267 & {$[0.00263,0.00271]$} & 0.00267 & 0.00267 \\
\hline 36 & 1956 & 0.00284 & 0.00284 & {$[0.00279,0.00288]$} & 0.00284 & 0.00281 \\
\hline 37 & 1957 & 0.00308 & 0.00308 & {$[0.00304,0.00313]$} & 0.00309 & 0.00311 \\
\hline 38 & 1958 & 0.00321 & 0.00321 & {$[0.00317,0.00325]$} & 0.00321 & 0.00317 \\
\hline 39 & 1959 & 0.00348 & 0.00348 & {$[0.00344,0.00352]$} & 0.00348 & 0.00347 \\
\hline 40 & 1960 & 0.00382 & 0.00382 & {$[0.00378,0.00386]$} & 0.00382 & 0.00373 \\
\hline 41 & 1961 & 0.00404 & 0.00404 & {$[0.00400,0.00408]$} & 0.00404 & 0.00400 \\
\hline 42 & 1962 & 0.00459 & 0.00459 & {$[0.00454,0.00463]$} & 0.00459 & 0.00452 \\
\hline 43 & 1963 & 0.00503 & 0.00503 & {$[0.00498,0.00508]$} & 0.00504 & 0.00490 \\
\hline 44 & 1964 & 0.00534 & 0.00534 & {$[0.00530,0.00539]$} & 0.00534 & 0.00527 \\
\hline 45 & 1965 & 0.00591 & 0.00591 & {$[0.00586,0.00596]$} & 0.00591 & 0.00583 \\
\hline 46 & 1966 & 0.00638 & 0.00637 & {$[0.00633,0.00642]$} & 0.00638 & 0.00625 \\
\hline 47 & 1967 & 0.00695 & 0.00695 & {$[0.00690,0.00701]$} & 0.00696 & 0.00686 \\
\hline 48 & 1968 & 0.00772 & 0.00772 & {$[0.00766,0.00777]$} & 0.00772 & 0.00765 \\
\hline 49 & 1969 & 0.00853 & 0.00853 & {$[0.00847,0.00859]$} & 0.00853 & 0.00847 \\
\hline 50 & 1970 & 0.00903 & 0.00903 & {$[0.00897,0.00908]$} & 0.00902 & 0.00906 \\
\hline 51 & 1971 & 0.00981 & 0.00981 & {$[0.00975,0.00987]$} & 0.00982 & 0.00988 \\
\hline 52 & 1972 & 0.01049 & 0.01049 & {$[0.01043,0.01056]$} & 0.01049 & 0.01061 \\
\hline 53 & 1973 & 0.01131 & 0.01131 & {$[0.01124,0.01137]$} & 0.01131 & 0.01145 \\
\hline 54 & 1974 & 0.01223 & 0.01223 & {$[0.01216,0.01230]$} & 0.01223 & 0.01240 \\
\hline 55 & 1975 & 0.01323 & 0.01323 & {$[0.01316,0.01331]$} & 0.01324 & 0.01321 \\
\hline 56 & 1976 & 0.01421 & 0.01421 & {$[0.01413,0.01429]$} & 0.01421 & 0.01403 \\
\hline 57 & 1977 & 0.01487 & 0.01487 & {$[0.01479,0.01495]$} & 0.01486 & 0.01492 \\
\hline 58 & 1978 & 0.01608 & 0.01608 & {$[0.01600,0.01617]$} & 0.01609 & 0.01609 \\
\hline 59 & 1979 & 0.01706 & 0.01706 & {$[0.01697,0.01715]$} & 0.01706 & 0.01709 \\
\hline 60 & 1980 & 0.01850 & 0.01850 & {$[0.01840,0.01860]$} & 0.01850 & 0.01861 \\
\hline 61 & 1981 & 0.01981 & 0.01981 & {$[0.01971,0.01992]$} & 0.01981 & 0.01978 \\
\hline 62 & 1982 & 0.02085 & 0.02084 & {$[0.02074,0.02096]$} & 0.02084 & 0.02078 \\
\hline 63 & 1983 & 0.02269 & 0.02269 & {$[0.02258,0.02281]$} & 0.02270 & 0.02272 \\
\hline 64 & 1984 & 0.02384 & 0.02384 & {$[0.02371,0.02396]$} & 0.02383 & 0.02377 \\
\hline 65 & 1985 & 0.02580 & 0.02580 & {$[0.02567,0.02594]$} & 0.02581 & 0.02526 \\
\hline 66 & 1986 & 0.02727 & 0.02727 & {$[0.02712,0.02742]$} & 0.02727 & 0.02699 \\
\hline 67 & 1987 & 0.02842 & 0.02842 & {$[0.02826,0.02858]$} & 0.02842 & 0.02825 \\
\hline 68 & 1988 & 0.03025 & 0.03025 & {$[0.03007,0.03043]$} & 0.03025 & 0.03029 \\
\hline 69 & 1989 & 0.03225 & 0.03225 & {$[0.03206,0.03245]$} & 0.03226 & 0.03244 \\
\hline 70 & 1990 & 0.03452 & 0.03452 & {$[0.03431,0.03473]$} & 0.03452 & 0.03477 \\
\hline 71 & 1991 & 0.03683 & 0.03683 & {$[0.03660,0.03705]$} & 0.03683 & 0.03766 \\
\hline 72 & 1992 & 0.03942 & 0.03942 & {$[0.03916,0.03967]$} & 0.03941 & 0.03989 \\
\hline 73 & 1993 & 0.04276 & 0.04276 & {$[0.04248,0.04304]$} & 0.04277 & 0.04329 \\
\hline 74 & 1994 & 0.04507 & 0.04507 & {$[0.04477,0.04539]$} & 0.04506 & 0.04602 \\
\hline 75 & 1995 & 0.04927 & 0.04927 & {$[0.04893,0.04961]$} & 0.04927 & 0.04956 \\
\hline 76 & 1996 & 0.05364 & 0.05364 & {$[0.05327,0.05401]$} & 0.05366 & 0.05353 \\
\hline 77 & 1997 & 0.05762 & 0.05762 & {$[0.05721,0.05805]$} & 0.05762 & 0.05732 \\
\hline 78 & 1998 & 0.06313 & 0.06313 & {$[0.06267,0.06360]$} & 0.06312 & 0.06166 \\
\hline 79 & 1999 & 0.06878 & 0.06878 & {$[0.06827,0.06929]$} & 0.06879 & 0.06730 \\
\hline 80 & 2000 & 0.07477 & 0.07477 & {$[0.07417,0.07536]$} & 0.07476 & 0.07440 \\
\hline
\end{tabular}

Table 4.2: Mortality rates for generation aged 30 in 1950. 
Renshaw, A.E., \& Haberman, S. (2003a). Lee-Carter mortality forecasting with age specific enhancement. Insurance: Mathematics \& Economics 33, 255-272.

Renshaw, A., \& Haberman, S. (2003b). Lee-Carter mortality forecasting: a parallel generalized linear modelling approach for England and Wales mortality projections. Applied Statistics 52, 119-137.

Sithole, T.Z., Haberman, S., \& Verrall, R.J. (2000). An investigation into parametric models for mortality projections, with applications to immediate annuitants and life office pensioners' data. Insurance: Mathematics $\&$ Economics 27, 285-312.

Smith, A.F.M. \& Roberts, G.O. (1993). Bayesian computation via the Gibbs sampler and related Markov Chain Monte Carlo methods. Journal of the Royal Statistical Society - Series B $\mathbf{5 5}$. 